This item was submitted to Loughborough's Research Repository by the author.

Items in Figshare are protected by copyright, with all rights reserved, unless otherwise indicated.

Oscillations and patterns in spatially discrete models for developmental intercellular signalling

PLEASE CITE THE PUBLISHED VERSION

LICENCE

CC BY-NC-ND 4.0

REPOSITORY RECORD

Webb, Steven D., and Markus R. Owen. 2019. "Oscillations and Patterns in Spatially Discrete Models for Developmental Intercellular Signalling”. figshare. https://hdl.handle.net/2134/315. 


\title{
Oscillations and patterns in spatially discrete models for developmental intercellular signalling
}

\author{
Received: date / Revised version: date - (C) Springer-Verlag 2003
}

\begin{abstract}
We extend previous models for nearest neighbour ligand-receptor binding to include both lateral induction and inhibition of ligand and receptor production, and different geometries (strings of cells and hexagonal arrays, in addition to square arrays). We demonstrate the possibility of lateral inhibition giving patterns with a characteristic length scale of many cell diameters, when receptor production is included. In contrast, lateral induction combined with inhibition of receptor synthesis cannot give rise to a patterning instability under any circumstances. Interesting new dynamics include the analytical prediction and consequent numerical observation of spatiotemporal oscillations - this depends crucially on the production terms and on the relationship between the decay rates of ligand and free receptor.

Our approach allows for a detailed comparison with the model for Delta-Notch interactions of Collier et al. [4], and we find that a formal reduction may be made only when the ligand receptor binding kinetics are very slow. Without such very slow receptor kinetics, spatial pattern formation via lateral inhibition in hexagonal cellular arrays requires significant activation of receptor production, a feature that is not apparent from previous analyses.
\end{abstract}

\section{Introduction}

The development of spatial organisation is a fundamental requirement for the construction of every multicellular organism, from fruit flies to humans. A collection of cells must be able to arrange themselves in some way to form organs, limbs, digits, and so on. A key component of this process of organismal development is the determination of cell fate, whereby cells adopt a particular program of gene activation. Cell fates are regulated by a variety of mechanisms, typically mediated by the production and transport of signalling molecules which induce a response by binding to specific sites.

Steven D Webb: Department of Mathematical Sciences, Loughborough University, Loughborough, LE11 3TU, UK. e-mail: S.D.Webb@lboro.ac.uk

Markus R Owen: Department of Mathematical Sciences, Loughborough University, Loughborough, LE11 3TU, UK. e-mail: M.R.Owen@lboro.ac.uk.

Send offprint requests to: Markus R Owen

Key words: Delta-Notch - Juxtacrine - Lateral Inhibition - Morphogenesis 
Whilst experimentalists have been able to identify a large number of such proteins and their targets, it is difficult to determine how they act in concert to generate appropriate structures.

Mathematical and theoretical approaches have a long history in the effort to unravel this complex web of regulatory pathways. An important early contribution was made by Turing $[23,38]$, who showed that chemicals can react and diffuse in such a way that spatial patterns of concentration are established, consequently specifying cell fate. This mechanism has been applied to generate patterned solutions that are strikingly similar to natural animal coat patterns [30]. In contrast, Wolpert proposed that positional information is encoded by a gradient in morphogen concentration, which could be established by the diffusion of morphogen from a fixed source [43]. Cells would experience different levels of morphogen depending on their distance from the source, and adjust their behaviour accordingly. A number of potential morphogens that act in this fashion have been identified, but some modelling studies indicate that direct diffusion alone may not be able to set up appropriate gradients due to the binding of morphogen to its membrane receptors [15]. In contrast, Lander et al have recently shown that diffusive transport may in fact be effective when combined with complex interactions between receptors and the cell membrane [18]. A variety of other mathematical approaches have also been proposed [26,35].

Many patterns arising in early development have a very small spatial scale, which cannot be accounted for by continuum models such as those described above; for example, the fine-grained patterns in the eye of the fruit fly, Drosophila, illustrated in Figure 1(a). There is much evidence that a crucial role is played by direct signalling from a cell to its neighbour via membrane-bound proteins, called juxtacrine signalling. Such systems have the advantage that they are relatively well characterised experimentally a number of proteins and their receptors have been identified $[7,22]$. On the other hand, the analysis of such systems presents considerable mathematical challenges due to their spatially discrete nature.

The first model to consider juxtacrine signalling was formulated in terms of the activity of a protein and its receptor [4], incorporating a feedback loop termed lateral inhibition. The signalling molecule in this model is a protein called Delta which binds to the receptor Notch - this interaction is known to be important in early animal development [20,21,42]. The term lateral inhibition is used to describe a cell-cell interaction whereby a cell heading for a particular cell fate inhibits its neighbours from developing in the same way. In the Delta-Notch system, for example, high Delta expression in a cell downregulates Delta in its neighbours, via the receptors Notch on their cell surface $[16,20]$. This lateral inhibition has been shown to be a robust mechanism for the formation of spatial patterns - provided that the inhibition is sufficiently strong, small differences between neighbouring cells are self-amplifying, leading to the generation of fine grained patterns [4]. Detailed analysis, however, showed that in all cases the scale of the predicted patterns does not extend beyond a wavelength of two cells (for a linear array) 
or three cells (for a hexagonal array). Although this is consistent with the small spatial scale patterns in early animal development, longer wavelength patterns are observed; for example, during neuroblast segregation in the Drosophila embryo [36], in which the scale of the pattern is of the order of many cell diameters.

Recent evidence suggests that such longer wavelength patterns could be a result of the reverse of this mechanism, called lateral induction, in which receptor activation up-regulates ligand production [28,29,40,41]. Such induction is well established for some juxtacrine signals [34] including TGF- $\alpha$ and EGF binding to EGF-R [2,3] and the Delta-Notch system has also been shown to exhibit this up-regulation in some contexts [5,12,21,31]. Models that incorporate such up-regulation have been studied recently by Monk [25], Owen \& Sherratt [28] and Wearing et al [40,41]. The paper by Monk [25] was based on the approach of Collier et al [4], using the idea of protein activities to study the role of transforming growth factor- $\beta$ (TGF- $\beta$ ) in Xenopus mesodermal development. In the models of Owen \& Sherratt [28], and later Wearing et al $[40,41]$, the interacting variables are the numbers of signalling (ligand) molecules, and free and bound receptors on the cell surface which interact via elementary events, rather than the more arbitrary measure of protein activity. Figure 1(b) illustrates the binding of ligands on the surface of one cell to free receptors on an adjacent surface. The resulting bound receptors generate a signal which leads to induction or inhibition of ligand and receptor production. Intuitively, one would expect that lateral induction would smooth out any differences in Delta/Notch expression between neighbouring cells and lead to homogeneity in the tissue. However, Wearing et al [40] use the model of Owen \& Sherratt [28] to show that lateral induction is in fact a highly effective generator of spatial patterns, and in contrast to the mechanism studied by Collier et al [4], the Owen \& Sherratt [28] juxtacrine model can generate a wide range of long wavelength patterns.

In this paper, we extend the work of Wearing et al [40] to include both activation and inhibition of ligand and receptor production, and different geometries (strings of cells, and hexagonal arrays, in addition to square arrays). In Section 2, we introduce the model and describe the main assumptions. In Section 3, we use linear stability analysis similar to that used by Turing et al [38] to investigate the pattern forming potential of this system. We show that the conditions for pattern formation depend on the geometry of the cellular array, and on the intensity of the inhibition or induction that the cells experience. Interesting new dynamics also include the analytical prediction and consequent numerical observation of spatio-temporal oscillations. We discuss these solutions in Section 3.3. In Section 3.4, we calculate numerically the wavelength that corresponds to the fastest growing mode for different strengths of activation/inhibition in the model. This demonstrates that lateral inhibition can give instability to patterns with a characteristic length scale of many cell diameters, when receptor dynamics are included. In Section 4, we discuss the differences and similarities be- 
tween the models of Owen \& Sherratt [28] and that used by Collier et al [4], and we describe the conditions under which these two approaches are equivalent. This allows for a detailed comparison with the model for lateral inhibition in Delta/Notch by Collier et al [4]. The conclusions of our work are presented in Section 5.

\section{The mathematical model and juxtacrine averaging}

The model scheme, as presented in $[28,40]$, is as follows

$$
\begin{aligned}
& \frac{\partial a}{\partial t}=-k_{a} a\langle f\rangle+k_{d}\langle b\rangle-d_{a} a+P_{a}(b) \\
& \frac{\partial f}{\partial t}=-k_{a}\langle a\rangle f+k_{d} b-d_{f} f+P_{f}(b) \\
& \frac{\partial b}{\partial t}=k_{a}\langle a\rangle f-k_{d} b-k_{i} b .
\end{aligned}
$$

Here, $a(\mathbf{x}, t)$ is the number of ligand molecules, $f(\mathbf{x}, t)$ is the number of free receptors, and $b(\mathbf{x}, t)$ is the number of bound receptor-ligand complexes on the surface of a cell at time $t$ and position $\mathbf{x}$. We use a generic kinetic scheme for juxtacrine signalling similar to that of Waters et al [39] for EGF-EGF-R interactions: ligand on the surface of one cell binds reversibly, with binding constant $k_{a}$ and dissociation rate $k_{d}$, to free receptors on the surface of immediately neighbouring cells. The resulting bound receptors generate a signal which leads to activation or inhibition of ligand and receptor production. The newly synthesized ligand/receptors arrive at the cell surface at the rate $P_{a}(b)$ for ligand and $P_{f}(b)$ for receptor. They decay with rate constants $d_{a}$ and $d_{f}$, respectively, and the surface complexes are internalized with the rate constant $k_{i}$.

We restrict attention to the formation of patterns in a string of cells, or in two-dimensional sheets, which we represent as regular arrays of square or hexagonal cells. The \langle\rangle notation indicates the spatial coupling between the cells, and we call this the juxtacrine averaging term. For a line of cells indexed by the integers, $\mathbf{x}=j$, and

$$
\left\langle u_{j}\right\rangle=\frac{\left(u_{j-1}+u_{j+1}\right)}{2} .
$$

Here, and below, $u$ corresponds to $a, f$ or $b$, for each of the variables in (1). More generally, $\langle u\rangle=\frac{1}{N} \sum_{j^{\prime}} u_{j^{\prime}}$ where the sum is taken over the $N$ immediate neighbours of the cell. For a 2-d regular array of square cells with labelling $\mathbf{x}=(j, l)$,

$$
\left\langle u_{j, l}\right\rangle=\frac{u_{j, l-1}+u_{j, l+1}+u_{j-1, l}+u_{j+1, l}}{4},
$$


and for a 2-d array of hexagonal cells

$$
\left\langle u_{j, l}\right\rangle=\frac{u_{j, l+1}+u_{j+1, l+1}+u_{j+1, l}+u_{j-1, l}+u_{j-1, l-1}+u_{j, l-1}}{6} .
$$

These labelling schemes are illustrated in Figure 2. We also study the formation of striped patterns within two-dimensional square arrays. This was the focus of previous work in the case of lateral induction $[28,40,41]$, and is a subset of (3) in which cells in the same column are considered identical, so that $u_{j, l \pm 1}=u_{j, l}$.

\section{Linear stability analysis}

We wish to predict the pattern forming potential of this system by analysing the stability of the homogeneous steady state $\left(a_{e}, f_{e}, b_{e}\right)$ to spatially varying perturbations. We extend the earlier work on stripe formation and lateral induction to include different geometries and activation or inhibition of both ligand and receptor production. This allows for four combinations that we will characterise by the slopes of the production functions at a homogeneous steady state. Lateral inhibition has been well studied for the juxtacrine mode of communication in models formulated in terms of the activity of a protein and its receptor. However, this is the first time that inhibition has been studied in a system that reflects actual binding events, rather than simple protein activities. The linear analysis that we present is similar to that used by $[28,40]$, but we include the details here for completeness.

Linearizing about the homogeneous steady state by setting $a=a_{e}+\tilde{a}$, $f=f_{e}+\tilde{f}, b=b_{e}+\tilde{b}$, gives

$$
\begin{aligned}
& \frac{\partial \tilde{a}}{\partial t}=-k_{a} f_{e} \tilde{a}-k_{a} a_{e}\langle\tilde{f}\rangle+k_{d}\langle\tilde{b}\rangle-d_{a} \tilde{a}+\mathcal{A} b \\
& \frac{\partial \tilde{f}}{\partial t}=-k_{a}\langle\tilde{a}\rangle f_{e}-k_{a} a_{e} \tilde{f}+k_{d} \tilde{b}-d_{f} \tilde{f}+\mathcal{F} \tilde{b} \\
& \frac{\partial \tilde{b}}{\partial t}=k_{a}\langle\tilde{a}\rangle f_{e}+k_{a} a_{e} \tilde{f}-k_{d} \tilde{b}-k_{i} \tilde{b},
\end{aligned}
$$

where

$$
\mathcal{A}=P_{a}^{\prime}\left(b_{e}\right) \quad \text { and } \quad \mathcal{F}=P_{f}^{\prime}\left(b_{e}\right) .
$$

In this way, the strength of activation/inhibition is measured by its gradient at the homogeneous steady state. Thus activation (inhibition) of ligand production corresponds to $\mathcal{A}>0(\mathcal{A}<0)$, and activation (inhibition) of receptor production corresponds to $\mathcal{F}>0(\mathcal{F}<0)$.

We look for patterned solutions of the form $\tilde{u}=\bar{u} \exp (\alpha t+i \lambda \cdot \mathbf{x})$, where $\bar{u}$ is a constant, $\alpha$ is the growth rate of perturbations with wave number $\lambda$, and we consider an infinite lattice or periodic boundary conditions. For a 
one-dimensional string, $\mathbf{x}=j, \lambda$ is a scalar, and the juxtacrine averaging term becomes

$$
\left\langle\tilde{u}_{j}\right\rangle=\frac{\left(\tilde{u}_{j-1}+\tilde{u}_{j+1}\right)}{2}=\bar{u} e^{\alpha t+i \lambda j} \frac{\left(e^{-i \lambda}+e^{i \lambda}\right)}{2}=\bar{u} e^{\alpha t+\lambda j} \cos (\lambda) .
$$

Similar expressions can be obtained for the 2-d cases, for which $\lambda$ is a two component vector. Setting $\lambda_{1}$ and $\lambda_{2}$ to be the wavenumbers for the horizontal and vertical directions, we get for square cells

$$
\left\langle\tilde{u}_{j l}\right\rangle=\bar{u} e^{\alpha t+i \lambda \cdot \mathbf{x}} \frac{\left(\cos \left(\lambda_{1}\right)+\cos \left(\lambda_{2}\right)\right)}{2},
$$

and

$$
\left\langle\tilde{u}_{j l}\right\rangle=\bar{u} e^{\alpha t+i \lambda \cdot \mathbf{x}} \frac{\left(\cos \left(\lambda_{1}\right)+1\right)}{2}
$$

when the vertical wave number $\lambda_{2}=0$ in the case of striped patterns. For hexagonal cells

$$
\left\langle\tilde{u}_{j l}\right\rangle=\bar{u} e^{\alpha t+\lambda \cdot \mathbf{x}} \frac{\left(\cos \left(\lambda_{1}\right)+\cos \left(\lambda_{2}\right)+\cos \left(\lambda_{1}+\lambda_{2}\right)\right)}{3} .
$$

For notational simplicity, we define

$$
K(\lambda)= \begin{cases}\cos (\lambda) & \text { (1-d string) } \\
\frac{\cos \left(\lambda_{1}\right)+\cos \left(\lambda_{2}\right)}{2} & \text { (2-d square array) } \\
\frac{\cos \left(\lambda_{1}\right)+\cos \left(\lambda_{2}\right)+\cos \left(\lambda_{1}+\lambda_{2}\right)}{3} & \text { (hexagonal array) } .\end{cases}
$$

$K(\lambda)$ can be thought of as the "nearest neighbour contribution" to the equilibrium. In both the string and two-dimensional square arrays, $K(\lambda)$ is bounded between \pm 1 as $\lambda$ varies. However, $K(\lambda)$ is reduced to $[0,1]$ in the case of 2 -d striped patterns in square cells, and to $[-1 / 2,1]$ for hexagonal cells. From now on, we shall refer to these cases as (1L) for a 1-d string; (2S) for 2-d square arrays; (2Str) for 2-d striped patterns in square arrays; and $(2 \mathrm{H})$ for 2 -d arrays of hexagonal cells.

Substituting into the linearized model and dividing through by $\exp (\alpha t+$ $i \lambda \cdot \mathbf{x})$, the condition for non-trivial solutions gives the cubic characteristic 
equation $P(\alpha ; K(\lambda))=\alpha^{3}+a_{1} \alpha^{2}+a_{2}(K(\lambda)) \alpha+a_{3}(K(\lambda))$, where

$$
\begin{aligned}
a_{1} & =k_{a} a_{e}+k_{a} f_{e}+d_{a}+d_{f}+k_{d}+k_{i} \\
a_{2}(K(\lambda)) & =-K(\lambda)^{2} k_{a} f_{e}\left(k_{a} a_{e}+k_{d}\right)-K(\lambda) k_{a} f_{e} \mathcal{A}+\left(d_{a}+d_{f}\right)\left(k_{d}+k_{i}\right) \\
& +d_{a} d_{f}+k_{a} f_{e}\left(k_{a} a_{e}+d_{f}+k_{d}+k_{i}\right)+k_{a} a_{e}\left(d_{a}+k_{i}-\mathcal{F}\right), \\
a_{3}(K(\lambda)) & =-K(\lambda)^{2} k_{a} f_{e}\left(k_{a} a_{e}\left(k_{i}-\mathcal{F}\right)+k_{d} d_{f}\right)-K(\lambda) k_{a} d_{f} f_{e} \mathcal{A} \\
& +\left(k_{a} f_{e}+d_{a}\right)\left(k_{a} a_{e}\left(k_{i}-\mathcal{F}\right)+d_{f}\left(k_{d}+k_{i}\right)\right) .
\end{aligned}
$$

The roots of the characteristic equation determine the stability of the homogeneous steady state.

We consider where the homogeneous equilibrium is both stable to homogeneous perturbations, which corresponds to imposing $\lambda=\mathbf{0}$ (and gives $K=1$ in each of the 4 cases $(1 \mathrm{~L}),(2 \mathrm{~S}),(2 \mathrm{Str})$ and $(2 \mathrm{H}))$, and unstable to inhomogeneous perturbations. Note that the steady state $\left(a_{e}, f_{e}, b_{e}\right)$ is linearly stable to homogeneous perturbations if all the roots of $P(\alpha ; K=1)$ have $\operatorname{Re}(\alpha)<0$. For the steady state to be unstable to spatial disturbances we require at least one root of $P(\alpha ; K(\lambda))$ to have $\operatorname{Re}(\alpha(K(\lambda)))>0$ for some $\lambda \neq 0$.

\subsection{Stability to homogeneous perturbations}

We first reiterate the criteria derived in $[28,40]$ for the stability of the steady state to homogeneous perturbations. The Routh-Hurwitz conditions on $a_{1}$, $a_{2}(1)$ and $a_{3}(1)$ such that zeros of $P(\alpha ; K=1)$ have $\operatorname{Re}(\alpha)<0$ are $a_{1}>0$, $a_{3}(1)>0$ and $a_{1} a_{2}(1)-a_{3}(1)>0$. The coefficient $a_{1}$ is strictly positive and so condition one holds automatically. The other two conditions define two lines in $(\mathcal{A}, \mathcal{F})$ space which delimit the relevant regions. These are found by solving $a_{3}(1)=0$ and $a_{1} a_{2}(1)-a_{3}(1)=0$ to give

$$
\begin{aligned}
T_{1}: \quad \mathcal{F}= & k_{i}+\frac{d_{f}\left(k_{d}+k_{i}\right)}{k_{a} a_{e}}+\frac{d_{f} f_{e} k_{i}}{d_{a} a_{e}}-\frac{d_{f} f_{e}}{d_{a} a_{e}} \mathcal{A} \\
H_{1}: \quad \mathcal{F}= & k_{i}+d_{a}+\frac{d_{f} f_{e}}{a_{e}}+\frac{d_{a} d_{f}+\left(d_{a}+d_{f}\right)\left(k_{d}+k_{i}\right)}{k_{a} a_{e}} \\
& +\frac{d_{a}^{2}\left(d_{f}+k_{d}+k_{i}\right)+d_{a} k_{a}\left(d_{f} f_{e}+d_{a} a_{e}\right)+k_{a} k_{i} f_{e}\left(a_{1}-d_{f}\right)}{k_{a} a_{e}\left(a_{1}-d_{a}\right)} \\
& -\frac{f_{e}\left(a_{1}-d_{f}\right) \mathcal{A}}{a_{e}\left(a_{1}-d_{a}\right)} .
\end{aligned}
$$


These lines both have negative slope and intersect the $\mathcal{F}$-axis at positive values. The homogeneous steady state is stable if $\mathcal{F}$ lies below both of these lines, defining the region of stability. The relative slopes of these lines depend on the relationship between $d_{a}$ and $d_{f}$ : for $d_{f}<d_{a}$, the line $H_{1}$ has a steeper negative gradient than $T_{1}$; for $d_{f}>d_{a}$, the opposite is true; this has important implications for the prediction of oscillating patterns, which we will discuss later.

\subsection{Stability to inhomogeneous perturbations}

We now consider where the steady state is unstable for some $\lambda \neq \mathbf{0}$. We require at least one root of $P(\alpha ; K(\lambda))$ to have $\operatorname{Re}(\alpha(K(\lambda)))>0$. In other words, we require one of the Routh-Hurwitz conditions for the cubic polynomial $P(\alpha ; K(\lambda))$ to be violated. As we have seen, the spatial coupling between the cells means that $\lambda$ is mapped to $K \in[\kappa, 1)$, where $\kappa=-1$ for $(1 \mathrm{~L})$ and $(2 \mathrm{~S}), \kappa=0$ for $(2 \mathrm{Str})$, and $\kappa=-1 / 2$ for $(2 \mathrm{H})$. Since $a_{1}$ is strictly positive, instability can only arise when there exists some $K$ such that either $a_{3}(K)<0$ or $\Delta(K)=a_{1} a_{2}(K)-a_{3}(K)<0$. Note that a change in the sign of $a_{3}$ corresponds to a real eigenvalue crossing the imaginary axis. However, a change in the sign of $\Delta$ corresponds to a pair of complex conjugate eigenvalues crossing the imaginary axis with non-zero imaginary part (provided $a_{3} \neq 0$ ) - if $a_{3}>0$ this is a Hopf Bifurcation.

Both $a_{3}$ and $\Delta$ are quadratic in $K$, and positive at $K=1$ to ensure stability to homogeneous perturbations. They can only change sign via a double root at a minimum $K_{\mathrm{c}} \in[\kappa, 1$ ) (region 1 ); or via a single root at $\kappa$ (region 2). This classification can be found in Table 1, together with sketches illustrating these two transitions. The regions and the relevant bifurcations are derived in Appendix A, and summarised here, for each value of $\kappa$ corresponding to the different geometries.

Regions and bifurcations for $a_{3}(K)$.

- Region 1: $a_{3}(K)$ has a minimum at $K_{\mathrm{c}} \in[\kappa, 1)$ when $\mathcal{F}>\max \left\{R_{\kappa}, R_{1}\right\}$. $R_{\kappa}$ and $R_{1}$ are illustrated in Figure $3(\mathrm{a})$. The minimum value, $a_{3}\left(K_{c}\right)$, is less than zero when $\mathcal{F}>T_{d}^{+}$(see Figure $3(\mathrm{~b})$ ).

- Region 2: When $\mathcal{F}<\max \left\{R_{\kappa}, R_{1}\right\}, a_{3}(K)$ does not have a minimum in $[\kappa, 1)$, and a patterning bifurcation occurs via a single root $a_{3}(\kappa)=0$, along the line $T_{\kappa}$ (Figure $3(\mathrm{~b})$ ), with $a_{3}(\kappa)<0$ for $\mathcal{F}>T_{\kappa}$.

The regions where $a_{3}(K)<0$ for some $K \in[\kappa, 1)$ are shaded in Figure $3(\mathrm{c})$, subject to the constraint $\mathcal{F}<T_{1}$, for stability to homogeneous perturbations. The boundaries are the same for all geometries when $\mathcal{A}>0$. The light shading is for $\kappa=0$ : in this region patterns are predicted for stripes in arrays of square cells. For hexagons $(\kappa=-1 / 2)$ the region is larger, since $T_{-1 / 2}$ is steeper than $T_{0}$, and region 1 is larger for this value of $\kappa$ - the additional part has intermediate shading. For strings and square arrays $(\kappa=-1)$ the region is larger still (dark shading). This means that stripes in square arrays are predicted for the smallest region of parameter space. 
Regions and bifurcations for $\Delta(K)$.

- Region 1: $\Delta(K)$ has a minimum at $K_{\mathrm{c}} \in[\kappa, 1)$ when $\mathcal{F}<\min \left\{S_{\kappa}, S_{1}\right\}$. $S_{\kappa}$ and $S_{1}$ are illustrated in Figure 4(a). The minimum value, $\Delta\left(K_{c}\right)$, is less than zero when $\mathcal{F}$ lies between the two curves $H_{d}^{ \pm}$(see Figure 4(b)).

- Region 2: When $\mathcal{F}>\min \left\{S_{\kappa}, S_{1}\right\}, \Delta(K)$ does not have a minimum in $[\kappa, 1)$, and a patterning bifurcation occurs via a single root $\Delta(\kappa)=0$, along the line $H_{\kappa}$ (Figure $4(\mathrm{~b})$ ), with $\Delta(\kappa)<0$ for $\mathcal{F}>H_{\kappa}$.

We illustrate these regions in Figure 4(c), subject to $\mathcal{F}<H_{1}$, which is required for stability to homogeneous perturbations. The shading corresponds to different geometries in the same way as for Figure 3(c).

\subsection{Oscillating or stationary patterns?}

The two inequalities $a_{3}(K)<0$ and $\Delta(K)<0$ together define the region in the $(\mathcal{A}, \mathcal{F})$ plane where patterns may form - the union of the shaded regions from Figure 3(c) and Figure 4(c), below the two lines $T_{1}$ and $H_{1}$. Since $\Delta(K)=0$ corresponds to a Hopf bifurcation, the precise details of this union will determine whether oscillatory or stationary patterns are to be expected.

Recall that the homogeneous steady state is stable to homogeneous perturbations when $\mathcal{F}<T_{1}$ and $\mathcal{F}<H_{1}$. Within this region, the equilibrium is unstable to inhomogeneous perturbations for $\mathcal{F}>\min \left\{T_{d}^{+}, T_{\kappa}, H_{d}^{-}, H_{\kappa}\right\}$, where $\kappa=-1$ for strings/squares, $\kappa=-1 / 2$ for hexagons, and $\kappa=0$ for stripes in square arrays.

A qualitative illustration of these conditions for strings/squares is given in Figure 5 - pattern formation is possible in the shaded regions. Note that pattern formation is not predicted in the lower-right quadrant of the $(\mathcal{A}, \mathcal{F})$ plane, corresponding to activation of ligand and inhibition of receptor production. There are four possible configurations for the pattern region, according to the relative slopes of the lines $T_{-1}$ and $H_{-1}$, and the location of their point of intersection. For $d_{f}>d_{a}$, the line $T_{-1}$ is steeper than $H_{-1}$, and these two lines must intersect at a positive value of $\mathcal{F}>T_{0}$, while $\mathcal{A}$ at this point can take either sign. As illustrated in Figure 5(a) and (b) this means that any patterning bifurcation is via $a_{3}(K)=0$, and a Hopf bifurcation is not possible. For $d_{f}<d_{a}$, the line $H_{-1}$ is steeper than $T_{-1}$, and their intersection occurs for $\mathcal{F}<T_{0}$ and $\mathcal{A}<0$. Thus, there is a window of $\mathcal{A}$ and $\mathcal{F}$ where the steady state is unstable to patterns via a Hopf bifurcation, and limit cycle oscillations are predicted; this is the dark shaded region between the lines $T_{-1}$ and $H_{-1}$ in Figure 5(c) and (d).

The picture for hexagons is similar, except that the relative slopes of the two lines $T_{-1 / 2}$ and $H_{-1 / 2}$ depend upon $k_{a}$ and $f_{e}$ as well as $d_{a}$ and $d_{f}$. 
The slopes of these lines are given by

$$
\begin{aligned}
& \text { slope } T_{-1 / 2}=\frac{2 f_{e} d_{f}}{a_{e}\left(3 k_{a} f_{e}+4 d_{a}\right)}, \text { and } \\
& \text { slope } H_{-1 / 2}=\frac{2 f_{e}\left(k_{a} a_{e}+k_{a} f_{e}+d_{a}+k_{d}+k_{i}\right)}{a_{e}\left(k_{a} f_{e}+4 k_{a} a_{e}+4 d_{f}+4 k_{d}+4 k_{i}\right)} .
\end{aligned}
$$

For $d_{f}>d_{a}+3 k_{a} f_{e} / 4$, the line $T_{-1 / 2}$ is steeper than $H_{-1 / 2}$, these two lines must intersect for $\mathcal{F}>T_{0}$, and there can be no Hopf bifurcation. Conversely, for $d_{f}<d_{a}+3 k_{a} f_{e} / 4, H_{-1 / 2}$ is steeper than $T_{-1 / 2}$, their intersection is for $\mathcal{F}<T_{0}$, and there is again a window of $\mathcal{A}$ and $\mathcal{F}$ in which oscillations are predicted.

For our values of the kinetic parameters (see Appendix B), the slope of $T_{-1 / 2}$ is in the range $10^{-2}$ to $10^{-1}$, whereas the slope of $T_{-1}$ is $\mathrm{O}(1)$. This stems from the term $3 k_{a} f_{e}+4 d_{a}$ in (17a) which is very large compared to corresponding term, $d_{a}$, in the slope of $T_{-1}$. When there is no bifurcation to oscillations the lower boundary of the pattern region is therefore flatter for hexagons compared to strings/squares. This means patterns for hexagons require stronger ligand inhibition when receptor production is weakly activated (or inhibited). When $d_{f}<d_{a}+3 k_{a} f_{e} / 4$, however, the slope of $H_{-1 / 2}$ is comparable to the slopes of $T_{-1}$, and $H_{-1}$. Hence, for a weak (or inhibitory) receptor response, it is easier to get patterns for hexagons via a Hopf bifurcation. Note as well that, for our parameter sets, $d_{f}>d_{a}+3 k_{a} f_{e} / 4$ requires $d_{f}$ to be $\mathrm{O}(1)$, giving a turnover time for free receptor of about one minute - considerably faster than can be expected for most proteins.

In Figure 6, we track the bifurcations for a 2-cell system using AUTO [6]. We fix $\mathcal{F}=0$ and allow $\mathcal{A}$ to vary. The boundary conditions are periodic, which is equivalent to an infinite string in which alternate cells are identical. The two cases shown in Figure 6 correspond to moving along the line $\mathcal{F}=$ 0 in Figures 5(a) and (c), with $d_{f}>d_{a}$ and $d_{f}<d_{a}$, respectively. The homogeneous steady state becomes unstable as $\mathcal{A}$ decreases, and a nonuniform steady state branches out at the bifurcation values $\mathcal{A}=-0.215$ in (a) and $\mathcal{A}=-0.289$ in (b).

In Figure 6(a), stable (unstable) stationary solutions are solid (dashed). The non-uniform state is stationary, with one branch giving the equilibrium value $b_{1}$, say, and the other branch giving $b_{2}$. This gives a pair of heterogeneous steady states $\left(b_{1}, b_{2}\right)$ or $\left(b_{2}, b_{1}\right)$. Spatial perturbations of the unstable homogeneous steady state grow and the levels of bound receptors approach one of the pair of stable heterogeneous steady states. When an equilibrium is reached, one cell has high bound receptors, while the other cell has low bound receptors. When there are more than 2 cells in a line, the pattern is that of alternating high and low levels of bound receptor.

In Figure $6(\mathrm{~b})$, when $\mathcal{A} \approx-0.289, \Delta(K)=0$ and $a_{3}(K)>0$, so that there is a Hopf bifurcation. Solid (open) circles indicate stable (unstable) limit cycle oscillations for the two cell system, in which the levels 
of molecules on the two cells oscillate out of phase. As $\mathcal{A}$ is decreased further, however, a limit point is reached, at which periodic solutions are lost in favour of a stable heterogeneous steady state. Prior to this limit point, there is an interval of $\mathcal{A}$ where both these types of solution coexist - this behaviour arises from a subcritical Hopf bifurcation of the steady heterogeneous solution.

The qualitative nature of oscillatory patterns in larger systems of cells in 1-d can be seen in Figure 7. Numerical investigation demonstrates that two possible long term behaviours occur. The system can evolve to a standing wave, which is the natural extension of the behaviour observed in the 2 cell system - each cell oscillates out of phase with its neighbours, between high and low levels of bound receptors with every second cell identical (see Figure $7(\mathrm{a})$ ). However, different initial conditions can also lead to a travelling wave - as seen in Figure 7(b) (details of the parameter values and the particular form of the production functions $\left.P_{a}(b)\right)$ and $P_{f}(b)$ are given in Appendix B). It seems that the number of cells is important, as well as the initial data, and travelling waves are observed more frequently in larger arrays. However, it is not clear how these differences specifically drive the selection from standing to traveling waves, and a detailed analysis of this has yet to be carried out.

\subsection{Fastest growing modes}

When $\mathcal{A}$ and $\mathcal{F}$ are within the shaded region defined in Figure 5, perturbations of the homogeneous steady state diverge to a heterogeneous (patterned) solution. The wavelength of the observed patterns depends crucially on the production strengths $\mathcal{A}$ and $\mathcal{F}$. Previously the dispersion relation $P(\alpha)=\alpha^{3}+a_{1} \alpha^{2}+a_{2}(K) \alpha+a_{3}(K)=0$ has been studied when $\mathcal{A}, \mathcal{F}>0$ and $\alpha$ is small [40]. The nonlinear terms can then be neglected to give $\alpha \approx-a_{3}(K) / a_{2}(K)$, and a good approximation to $K$ corresponding to the largest possible $\alpha$ is given by $K_{\mathrm{c}}$ in (40). In contrast to earlier work by Collier et al [4], Wearing et al demonstrated that the juxtacrine model (1) can generate a wide range of pattern wavelengths.

We will now investigate the possibility of such long wavelength instabilities in the entire $(\mathcal{A}, \mathcal{F})$ space - including lateral inhibition of ligand production, as considered by Collier et al for Delta-Notch [4]. For values of $\mathcal{A}$ and $\mathcal{F}$ in the pattern region far from the bifurcation curves the assumption of small $\alpha$ may not be valid, and $K_{\mathrm{c}}$ may not be a good approximation to the fastest growing mode. Hence, although algebraically difficult, we solve the cubic $P(\alpha)=0$ numerically for each point $(\mathcal{A}, \mathcal{F})$ in the parameter space and estimate the $K$ which gives the root with the largest positive real part $\operatorname{Re}(\alpha)$.

Before considering each cell geometry in turn, we first discuss the relationships between the functions $K(\lambda)$, given by (11), and the corresponding integer wavelengths for regular patterns. For a string of cells $\lambda=2 \pi / \omega$; for squares and hexagons $\lambda_{1}=2 \pi / \omega_{1}$ and $\lambda_{2}=2 \pi / \omega_{2}$. These regular patterns correspond to a periodic modulation of cellular ligand and receptor 
expression in the epithelium, with a single peak per period. In contrast, non-integer rational $\omega$ also gives periodic patterns, but with multiple peaks per period. For example, $\omega=10 / 7$ gives patterns $u \propto \cos (14 \pi j / 10)$, for $u=a, f, b$, which has 3 peaks per period of 10 cells. We remark that there may be circumstances in which only such irregular modes are unstable, or that such modes are the fastest growing.

Figure 8 illustrates the relationship between wavelengths and $K$ for each geometry. For strings and 2-d striped patterns $\left(\lambda_{2}=0\right)$, each integer wavelength gives a unique $K$, and for strings the only negative values of $K$ are -1 and $-1 / 2$, corresponding to wavelengths $\omega=2$ and $\omega=3$. For squares and hexagons, integer wavelengths give a much finer gradation of $K$. In addition, different combinations of $\omega_{1}$ and $\omega_{2}$ give the same value of $K$, and different patterns can have the same linear growth rate (see Figure 8(c) and (d)). Note that the lowest value of $K$ for hexagons, $K=-1 / 2$, corresponds to wavelength 3 patterns in the $j$ and $l$ directions, $\omega_{1}=\omega_{2}=3$.

Figures 9 and 10 show the value of $K$ corresponding to the regular mode with the largest linear growth rate, as a function of $\mathcal{A}$ and $\mathcal{F}$. Areas that are not shaded are either unstable to homogeneous perturbations or stable to inhomogeneous perturbations. The fastest growing modes $K$ are indicated on the same grey scale as that used in Figure 8: for example, in a string of cells (part (a)), black indicates wavelength 2 cell patterns, intermediate shading is for patterns with wavelength 4 cells and light grey indicates longer $(>8)$ wavelengths. For all geometries and parameter sets, longer wavelengths (i.e. $K$ closest to 1) are predicted for the strongest ligand and weakest receptor production in the $\mathcal{A}, \mathcal{F}>0$ pattern region - this agrees with the trend previously predicted for stripe formation in square arrays [40]. Figure 9 shows the fastest growing regular mode when $d_{f}>d_{a}$, so that there is no bifurcation to an oscillatory solution in strings/squares. A surprising result is that, even for $\mathcal{A}<0$ (lateral inhibition), patterns of wavelength longer than 2 cells are possible. This occurs when there is sufficiently strong receptor activation and weak ligand inhibition - for example, the intermediate shaded area for strings in Figure 9(a) has a fastest growing integer mode of wavelength 4 . In fact, for all geometries, wavelengths of at least 4 cells are predicted when $K=0$. These longer wavelength patterns give way to short wavelengths when the inhibition in ligand production is increased (i.e. $\mathcal{A}$ more negative).

Note that the pattern region for hexagons is smaller than for a string or squares. It appears in Figure 9(c) that the pattern region is bounded by a horizontal line, but in fact it is defined by the diagonal line $T_{-1 / 2}$, which has a very shallow positive slope in comparison to the line $T_{-1}$. The line $H_{-1 / 2}$ is in fact steeper than the line $T_{-1 / 2}$ for this parameter set, so that there are bifurcations to oscillations for hexagons. However, these oscillations first occur around $\mathcal{A}=-0.5$, giving a very large negative Hill coefficient of $m=-50$, which is far more negative than can be expected biologically. In contrast, oscillations in strings and squares are possible when 
$m$ is around -5 . In general, patterns for hexagons are hard to obtain when ligand binding induces weak or inhibitory receptor production.

Figure 10 shows the case when $d_{f}<d_{a}$ - part (a) for a string, (b) for squares and (c) for hexagons. Recall that oscillating patterns are predicted in the area below the line $T_{\kappa}$ and above the line $H_{\kappa}$, with $\kappa=-1$ for strings/squares and $\kappa=-1 / 2$ for hexagons. This analysis predicts that bifurcations to oscillating solutions are always for a wavelength of 2 cells in a string/squares, and 3 cells in hexagons. This type of oscillation is indeed what we see for a string in Figure 7.

Figure 11 shows the results of 3 different simulations of the model (1), for a string, squares and hexagons. We choose $d_{f}>d_{a}$ so that there is no bifurcation to an oscillating solution. Each simulation is for varying strengths of lateral inhibition and receptor production. This corresponds to different locations in the left half plane $(\mathcal{A}<0)$ of the $(\mathcal{A}, \mathcal{F})$ parameter spaces in Figure 9. In each case, the boundary conditions are periodic, and the initial conditions are small random perturbations about the homogeneous steady state. The parameter values used are given in Appendix B and the figure legend. These simulations confirm the trends in pattern wavelength predicted by linear analysis. In particular, longer wavelengths are generated even with inhibition of ligand production - in contrast to the short wavelength patterns predicted by Collier et al [4]. Lateral induction in ligand production $(\mathcal{A}>0)$ has been investigated previously in the case of striped patterns $[28,29,40,41]$, showing a wide range of long pattern wavelengths. Similar features are also observed for squares and hexagons, but we omit these results for brevity.

Only in cases (a) and (d) do we see regular solutions with a wavelength matching that predicted. In general, random initial conditions give mixed solutions with no regular form of pattern. Moreover, as the initial conditions vary, the final pattern also varies - for example, perturbing a single cell or a line of cells seems to generate more regular patterns. Linear analysis is a good predictor for the onset of a patterning instability, and, although the patterns seen in our simulations have some irregularities, they demonstrate good qualitative agreement with the linear analysis, showing the correct trend in wavelength. The lack of quantitative agreement means that the nonlinearities in the model must overide the wavelengths predicted by linear analysis. Furthermore, linear analysis only predicts pattern formation, but does not give necessary conditions for the existence of inhomogeneous solutions - such solutions may exist outside the region of linear instability. The full nonlinear problem has been investigated in a 2-cell system for striped patterns [41], but a thorough understanding of nonlinear pattern selection on larger arrays and for different cell geometries has yet to be carried out.

\section{Relation to the model of Collier et al. [4]}

Nearest neighbour signalling has previously been investigated by Collier et al [4]. Their model incorporates a feedback loop in which receptor activation 
down-regulates ligand production - the ligand being Delta in Drosophila which binds to the receptor Notch on adjacent cells. The Collier et al model in non-dimensional form is given by

$$
\begin{aligned}
& \frac{d D}{d t}=v\{g(N)-D\} \\
& \frac{d N}{d t}=f(\langle D\rangle)-N,
\end{aligned}
$$

where $N(\mathbf{x}, t)$ denotes the level of Notch activation in a cell, reflecting the level of inhibition that cell experiences from its neighbours, and $D(\mathbf{x}, t)$ denotes the level of Delta activity, reflecting the amount of inhibition that it delivers to its neighbours. $f(\langle D\rangle)$ is an increasing function representing the rate of production of Notch activity in response to increasing amounts of Delta activity in neighbouring cells. $g(N)$ models lateral inhibition, so that the rate of production of Delta activity is a decreasing function of the level of activated Notch in the same cell. The production of Delta and Notch activity is balanced by linear decay terms.

The functions $f$ and $g$ used in Collier et al are defined as

$$
f(D)=\frac{A D^{k}}{B+D^{k}}, \quad g(N)=\frac{1}{1+C N^{h}} .
$$

where $A, B, C>0$ and $h \geq 1$. Monovalent ligand binding is given by $k=1$, and co-operative binding is implicitly represented by $k \geq 2$. An example of a co-operative reaction between ligand and receptor is when the receptor has more than one binding site, so that after binding to a ligand molecule a single receptor molecule can then bind to more ligand molecules. For example, the $\mathrm{IP}_{3}$ receptor, a $\mathrm{Ca}^{2+}$ channel located in the endoplasmic reticulum of a cell, has one binding site for the messenger $\mathrm{IP}_{3}$ and two binding sites for calcium. Each nicotinic receptor has two binding sites for acetylcholine, one of a group of biochemicals known as neurotransmitters that carry nerve impulses from one neuron to another. The estrogen receptor dimerizes and exhibits cooperative ligand binding as part of its normal functioning [1]. Another important co-operative behaviour is when the ligand is multivalent, such as platelet derived growth factor (PDGF) which can cross-link and bind to two or more receptors $[9,11]$. Tumour necrosis factor- $\beta$ is a trimeric ligand that binds simultaneously to three receptor molecules [19]. In other systems, monovalent ligands that are incapable of crosslinking two or more receptors can still induce receptor clustering. For example, acidic fibroblast growth factor (aFGF) is itself incapable of inducing dimerization of its receptor but forms a multivalent complex with heparan sulfate proteoglycans that can in turn bind to two or more receptors [37]. Binding of epidermal growth factor (EGF) to solubilized EGF receptors also causes rapid receptor dimerization [8]. All these types of interaction can lead to receptor "activity" qualitatively similar to $f(x)$ with $k>1$. 
It was shown that, given sufficiently strong feedback, $(18,19)$ can generate patterns with a length scale of two cells (for a string) or three cells (for hexagons), in which cells with a high level of Delta expression (and low Notch) are surrounded by cells with low Delta (and high Notch) [4]. This is consistent with some fine-grained patterns observed in early development. This model was the first to consider Juxtacrine signalling, but it is very different from (1) because it is formulated in terms of the activity of a protein and its receptor, rather than specific binding events. The aim of this section is to investigate the relationship between these two approaches and study how our model (1) can reduce to that studied by Collier et al, thereby allowing for a detailed comparison.

We begin with the monovalent case described by the kinetic scheme in (1), so that the receptor has only one binding site. First, we make the assumption that there is a fixed total number of receptors, $f_{0}$, on the surface of each cell, so that $f+b=f_{0}$ for all $\mathbf{x}$. Then (1) reduces to just two equations for each cell, for $a$ and $b$. Such a restriction means that the rate of free receptor production must equal the rate of internalization of bound receptors plus the rate of free receptor decay, so that

$$
P_{f}(b)=k_{i} b+d_{f} f=\left(k_{i}-d_{f}\right) b+d_{f} f_{0} .
$$

After a little re-arranging of the $b$ equation (1c), we then get

$$
\begin{aligned}
& \frac{\partial a}{\partial t}=-k_{a} a\left(f_{0}-\langle b\rangle\right)+k_{d}\langle b\rangle-d_{a} a+P_{a}(b) \\
& \frac{\partial b}{\partial t}=[R(\langle a\rangle)-b]\left\{k_{i}+k_{d}+k_{a}\langle a\rangle\right\},
\end{aligned}
$$

where

$$
R(\langle a\rangle)=\frac{k_{a} f_{0}\langle a\rangle}{k_{d}+k_{i}+k_{a}\langle a\rangle} .
$$

This reduced model $(21,22)$ and Collier et al's model $(18,19)$ have the same homogeneous steady states (so that $\langle u\rangle=u$ ), provided $k_{i}=0$ and

$$
k=1, \quad A=f_{0}, \quad B=\frac{k_{i}+k_{d}}{k_{a}}, \quad v=d_{a}, \quad g(\cdot)=\frac{P_{a}(\cdot)}{d_{a}} .
$$

If the binding, dissociation and internalization terms in (21) are small in comparison to the decay and production terms $d_{a} a$ and $P_{a}(b)$, we may write $k_{a}=\varepsilon k_{a}^{*}, k_{d}=\varepsilon k_{d}^{*}$ and $k_{i}=\varepsilon k_{i}^{*}$. In the limit as $\varepsilon \rightarrow 0$ the two models have the same homogeneous and inhomogeneous steady states, although the models are dynamically different due to the multiplicative factor $\left\{k_{i}+k_{d}+\right.$ $\left.k_{a}\langle a\rangle\right\}$ in (21b).

In this way $b$ can be thought of as being similar to the Notch activation variable $N$ in the Collier et al model, and $a$ as similar to the level of Delta activity $D$. The function $R(\langle a\rangle)$ is a Monod type Hill function describing the 
increase in Notch activity due to monovalent binding by Delta in neighbouring cells, and $P_{a}(b)=d_{a} g(b)$ (with $h>0$ ) describes how receptor activation down-regulates ligand (Delta) production.

We now compare the conditions for pattern formation in the two systems when $k_{a}, k_{d}$ and $k_{i}$ are not small. Collier et al have shown that the homogeneous steady state $\left(D_{e}, N_{e}\right)$ is linearly stable if

$$
f^{\prime}\left(D_{e}\right) g^{\prime}\left(N_{e}\right)<1,
$$

which is always satisfied for lateral inhibition $\left(g^{\prime}<0\right)$. Instability to inhomogeneous perturbations is given by

$$
f^{\prime}\left(D_{e}\right) g^{\prime}\left(N_{e}\right)<\frac{1}{\kappa},
$$

where $\kappa=-1$ for strings/squares, and $\kappa=-1 / 2$ for hexagons. We will now look for the corresponding conditions in our reduced model $(21,22)$. We linearize about the homogeneous steady state $\left(a_{e}, b_{e}\right)$ in the usual way, and look for solutions proportional to $\exp (i \lambda \cdot \mathbf{x})$, where $\lambda$ is the wave number. The stability matrix at the homogeneous steady state is then

$$
J(K)=\left(\begin{array}{cc}
-k_{a}\left(f_{0}-b_{e}\right)-d_{a} & \mathcal{A}+K\left(k_{a} a_{e}+k_{d}\right) \\
k_{a}\left(f_{0}-b_{e}\right) K & -k_{a} a_{e}-k_{d}-k_{i}
\end{array}\right),
$$

where $\mathcal{A}=P_{a}^{\prime}\left(b_{e}\right)$ again represents the strength of activation/inhibition of ligand production, and $K$ is given by (11) in Section 2. Linear stability to homogeneous perturbations $(\lambda=\mathbf{0} \Leftrightarrow K=1)$, is guaranteed if trace $(J(1))$ is negative and $\operatorname{det}(J(1))$ is positive. The first condition is automatically satisfied since $\operatorname{trace}(J)$ is strictly negative. The second condition requires

$$
\mathcal{A}<\mathcal{A}_{\text {hom }}=k_{i}+\frac{d_{a}\left(k_{a} a_{e}+k_{d}+k_{i}\right)}{k_{a}\left(f_{0}-b_{e}\right)},
$$

which is satisfied in the case of lateral inhibition $(\mathcal{A}<0)$.

Considering inhomogeneous perturbations, it is clear that trace $(J(K))<$ 0 for $K \in[\kappa, 1)$, so a patterning instability must arise through a change in sign from positive to negative of $\operatorname{det}(J(K))$, and any unstable mode must have a real positive eigenvalue. Now $\operatorname{det}(J(K))$ is a quadratic function of $K$ with a negative coefficient of $K^{2}$, so it has a maximum. Since stability to homogeneous perturbations requires $\operatorname{det}(J(1))>0, \operatorname{det}(J(K))$ can only be negative for some $K \in[\kappa, 1)$ if it is negative at $K=\kappa$. In addition, it must follow that $\operatorname{det}(J(K))>\operatorname{det}(J(\kappa))$ for all $K \in(\kappa, 1)$. The largest eigenvalue of $J(K)$ is given by the most negative value of $\operatorname{det}(J(K))$, so the fastest growing mode is always given by $K=\kappa$, giving a wavelength of 2 for strings/squares and 3 for hexagons.

The bifurcation when $\operatorname{det}(J(-1))=0$ occurs at the point

$$
\mathcal{A}_{-1}^{*}=-\mathcal{A}_{\text {hom }}=-k_{i}-\frac{d_{a}\left(k_{a} a_{e}+k_{d}+k_{i}\right)}{k_{a}\left(f_{0}-b_{e}\right)},
$$


and likewise $\operatorname{det}(J(-1 / 2))=0$ gives

$$
\mathcal{A}_{-1 / 2}^{*}=-2 k_{i}-\frac{2 d_{a}\left(k_{a} a_{e}+k_{d}+k_{i}\right)}{k_{a}\left(f_{0}-b_{e}\right)}-\frac{3}{2}\left(k_{a} a_{e}+k_{d}\right)<\mathcal{A}_{-1}^{*} .
$$

The homogeneous steady state becomes unstable when $\mathcal{A}<\mathcal{A}_{\kappa}^{*}$, for $\kappa=$ $-1,-1 / 2$. Note that $\mathcal{A}_{-1 / 2}^{*}<\mathcal{A}_{-1}^{*}$, so stronger inhibition is required for patterns in hexagonal cells than for a string or squares, as was also found in Collier et al [4] — see equation (25). Interestingly, patterns for our reduced model $(21,22)$ are predicted when

$$
R^{\prime}\left(b_{e}\right) \mathcal{A}<R^{\prime}\left(b_{e}\right) A_{\kappa}^{*}=\frac{d_{a}}{\kappa}+\frac{k_{a} f_{0}\left(k_{d}+k_{i}\right)}{k_{a} a_{e}+k_{d}+k_{i}} \frac{1-\kappa^{2}}{\kappa},
$$

which, with the identification outlined in (23), corresponds precisely to (25) when $\kappa=-1$ (strings/squares). However, the second term does not disappear when $\kappa=-1 / 2$ (hexagons), so we see that the missing binding and dissociation terms in the Collier et al model make a significant difference to the conditions for pattern formation in hexagonal arrays - an order of magnitude for the biologically motivated parameter values we have considered (see Appendix B).

The assumption that the total number of receptors on each cell is fixed yields $P_{f}^{\prime}\left(b_{e}\right) \equiv \mathcal{F}=k_{i}-d_{f}$. With $d_{f}$ fixed, we can vary $k_{i}$ to vary $\mathcal{F}$ in the reduced model and view the predictions of the linear analysis graphically in the $(\mathcal{A}, \mathcal{F})$ plane. The points $(\mathcal{A}, \mathcal{F})=\left(\mathcal{A}_{-1}^{*}, k_{i}-d_{f}\right)$ and $\left(\mathcal{A}_{-1 / 2}^{*}, k_{i}-\right.$ $d_{f}$ ) lie on the lines $T_{-1}$ and $T_{-1 / 2}$, which delimit the regions for pattern formation for strings/squares and hexagons. Similarly, the point $\left(\mathcal{A}_{h o m}, k_{i}-\right.$ $d_{f}$ ) lies on the line $T_{1}$, which defines the region of stability to homogeneous perturbations. Thus, varying $k_{i}$ is equivalent to taking horizontal slices in the bifurcation diagram illustrated in Figure $3 . \mathcal{F}=k_{i}-d_{f}$ is clearly smaller than $\mathcal{F}=k_{i}+d_{f} k_{d} / k_{a} a_{e}$ which is the horizontal line tangent to the lowest part of the curve $T_{d}$, so that such horizontal slices can only be taken in the region where numerical analysis in Section 3.4 predicts pattern formation of wavelength 2 cells. Limit cycle oscillations in the full model are predicted for appropriate parameter values, but are not possible in the 2-variable system $(21,22)$ since the trace of $J$ is strictly negative. The reduction to two equations can therefore lead to the loss of solution types which are present in the full system.

We now consider an example of cooperative phenomena in the case when a receptor has two binding sites. A model for this consists of ligand $a$ on one cell that binds receptors $f$ on a neighbouring cell, to form a single bound complex $b$. This complex can combine with another ligand molecule to form a dual bound ligand-receptor complex $c$ :

$$
a+f \underset{k_{d}}{\stackrel{k_{a}}{\rightleftharpoons}} b, \quad a+b \underset{k_{-c}}{\stackrel{k_{c}}{\rightleftharpoons}} c .
$$


This $c$ generates a signal which leads to altered ligand and receptor production. In addition, both single and double complexes can be internalized with rates $k_{i}^{b}$ and $k_{i}^{c}$. Applying the law of mass action gives

$$
\begin{aligned}
& \frac{\partial a}{\partial t}=-k_{a} a\langle f\rangle+\left(k_{d}-k_{c} a\right)\langle b\rangle+k_{-c} c-d_{a} a+P_{a}(c) \\
& \frac{\partial f}{\partial t}=-k_{a}\langle a\rangle f+k_{d} b-d_{f} f+P_{f}(c) \\
& \frac{\partial b}{\partial t}=\left(k_{a} f-k_{c} b\right)\langle a\rangle-\left(k_{d}+k_{i}^{b}\right) b+k_{-c} c \\
& \frac{\partial c}{\partial t}=k_{c}\langle a\rangle b-\left(k_{-c}+k_{i}^{c}\right) c .
\end{aligned}
$$

We follow the same approach as in the monovalent case and reduce this system to a 2-variable model, for $a$ and $c$. We will then discuss how the multivalent case compares with that of Collier et al.

We can eliminate $f$ again by assuming a constant level of receptor expression, so that $f=f_{0}-b-c$. This again fixes receptor production so that

$$
P_{f}(c)=d_{f}\left(f_{0}-b-c\right)+k_{i}^{b} b+k_{i}^{c} c .
$$

We then apply a quasi-steady state assumption to equation (32c) for the intermediate single bound complex $b$. This condition can be justified when the intermediate $b$ is relatively short lived. This gives $b$ in terms of $c$ and $a$ :

$$
b=\frac{k_{a}\left(f_{0}-c\right)\langle a\rangle+k_{-c} c}{\left(k_{a}+k_{c}\right)\langle a\rangle+k_{d}+k_{i}^{b}} .
$$

Substituting this into (32d) and re-arranging gives

$$
\begin{gathered}
\frac{\partial a}{\partial t}=-k_{a} a\left(f_{0}-\langle b\rangle-\langle c\rangle\right)+\left(k_{d}-k_{c} a\right)\langle b\rangle \\
+k_{-c} c-d_{a} a+P_{a}(c) \\
\frac{\partial c}{\partial t}=\left[R_{2}(\langle a\rangle)-c\right]\left\{\alpha_{2}+\alpha_{3}\langle a\rangle+\alpha_{4}\langle a\rangle^{2}\right\},
\end{gathered}
$$

where

$$
\begin{gathered}
R_{2}(\langle a\rangle)=\frac{\alpha_{1}\langle a\rangle^{2}}{\alpha_{2}+\alpha_{3}\langle a\rangle+\alpha_{4}\langle a\rangle^{2}}, \\
\alpha_{1}=k_{a} k_{c} f_{0}, \quad \alpha_{2}=\left(k_{-c}+k_{i}^{c}\right)\left(k_{d}+k_{i}^{b}\right), \\
\alpha_{3}=k_{-c} k_{a}+k_{i}^{c}\left(k_{a}+k_{c}\right), \quad \alpha_{4}=k_{a} k_{c},
\end{gathered}
$$

and $b$ is given by (34). Again, if we assume that the binding and dissociation rates $k_{a}, k_{d}, k_{c}$ and $k_{-c}$ are small, so that the dominant mechanisms in the 
$a$ equation are ligand production $P_{a}(c)$ and decay $-d_{a} a$, then (35) reduces to a system that is similar to $(18,19)$. The level of Delta activity is again represented by $a$, and the level of Notch activity by $c$. However, because of the term $\alpha_{3}\langle a\rangle$ in (36), there is no straightforward formal identification between $f(\cdot)$ and $R_{2}(\cdot)$. Furthermore, the requirement for small binding and dissociation rates is not compatible with the quasi-steady state assumption on $b$, which in contrast requires fast binding and dissociation. If we are only concerned with steady states, then there is no need for a quasi-steady state argument, but the $\alpha_{3}\langle a\rangle$ term still means there is no direct equivalence between the steady states of $(32)$ and the Collier et al model $(18,19)$.

Multivalent reactions are often characterised by the Hill function, $f(x)=$ $A x^{k} /\left(B+x^{k}\right)$, as used by Collier et al [4]. The binding rate $R_{2}(\langle a\rangle)$ gives a similar sigmoidal response, but the $\alpha_{3}\langle a\rangle$ term in its denominator means that the Hill form gives the correct qualitative, but not quantitative, response. Another common approach is to assume that the two binding steps are telescoped into a single tri-molecular reaction $k a+f \rightleftharpoons c$. The binding rate (corresponding to Notch activity) in this case is given by

$$
R(\langle a\rangle)=\frac{k_{a} f_{0}\left\langle a^{k}\right\rangle}{k_{d}+k_{i}+k_{a}\left\langle a^{k}\right\rangle},
$$

which is a Hill function of $\left\langle a^{k}\right\rangle$ but not $\langle a\rangle^{k}$.

In summary, the qualitative form for the rate of receptor binding may make some difference to the details of a patterning instability, but more important is the inclusion or otherwise of binding and dissociation terms in the ligand equation (21a) or (35a). We have shown that these terms make a significant difference to the conditions for pattern formation, particularly in the case of hexagonal arrays.

\section{Discussion}

We have investigated a discrete mathematical model for nearest neighbour signalling in early development. The first model to consider such juxtacrine signalling was formulated by Collier et al [4] in terms of the activity of a protein and its receptor, and incorporated the phenomenon of lateral inhibition - where receptor binding down-regulates ligand expression. However, this model was unable to account for long range patterns, and predicted only wavelengths of two or three cells. Other work has highlighted the surprising result that lateral induction - where nearest neighbour binding up-regulates the production of new ligand and receptor - can generate a wide range of long wavelength patterns, whose characteristic scale can be the order of many cell diameters $[28,29,40,41]$.

In this paper, we have extended the work of Owen \& Sherratt $[28,29]$ and Wearing et al [40], to include both lateral induction and inhibition in ligand and receptor production, thereby allowing for a detailed comparison with the earlier work of Collier et al [4]. Analysis and numerical simulation 
demonstrates another surprising result: even with lateral inhibition in ligand production, patterns of wavelength longer than two or three cells are predicted. It has been suggested recently that lateral inhibition must be combined with other mechanisms to give longer wavelength patterns, such as cell growth [4], long-range filapodial contacts [24], or paracrine signalling $[17,33]$. However, we have shown that lateral inhibition can be sufficient by itself to predict longer-range patterns without the need for additional long range effects. It is important to realise that whilst linear analysis correctly predicts the formation of inhomogeneous solutions, in many cases it is not an accurate predictor of details such as wavelength. This has been addressed in some detail for striped patterns [41], but a thorough understanding of nonlinear pattern selection, including initiation by waves, is a crucial focus for future research $[27,32]$.

Other interesting new dynamics include the analytical prediction and consequent numerical observation of temporally oscillating patterns, taking the form of either standing waves or travelling waves. The generation of these patterns depends crucially on ligand and receptor production, and on the relationship between the decay rates of ligand and free receptor $\left(d_{a}\right.$ and $d_{f}$, respectively). When $d_{f}<d_{a}$ ( $d_{f}<d_{a}+3 k_{a} f_{e} / 4$ for hexagons), and with lateral inhibition of ligand production, the homogeneous steady state can become unstable to inhomogeneous perturbations via a Hopf bifurcation, and limit cycle oscillations are predicted.

Oscillating patterns have been characterized during mesoderm development. For instance, mRNAs for Notch signalling molecules such as the bHLH factor Hes1 oscillate with 2-hour cycles during somite segmentation [10]. In early zebrafish development, Delta $\mathrm{C}$ shows oscillating expression [13]. In chick and mouse, expression of the Notch modulator Lunatic Fringe oscillates [14]. The chick genes c-hairy 1 and c-hairy 2 are also expressed in a cyclic fashion, similar to that observed for Hes1 mRNA during somitogenesis [14]. The model we have described in this paper is generic for juxtacrine communication, and the timescale of the oscillations implied by our kinetic parameters may be inappropriate for these particular examples. The specific application of this model to the somite segmentation clock is a natural extension of this work. This research could provide valuable insight into how the temporal periodicity of the clock modulates Delta/Notch signalling in the mesoderm in order to produce the spatial periodicity of the somites.

The approaches of Owen \& Sherratt [28] and Collier et al [4] are very different: Owen \& Sherratt's approach was to develop a three-variable model reflecting the elementary binding events of signalling molecules (ligands) binding to free receptors, in contrast to the more arbitrary measures of protein activity used in the two-variable model of Collier et al. Furthermore, in Collier et al, two-dimensional patterns were presented on hexagonal arrays, whereas Owen \& Sherratt [28] and Wearing et al [40] only considered striped patterns on a square grid. In this paper, we have addressed these differences, and have identified the conditions under which the two models are equivalent. Specifically, we have shown that with low affinity binding 
and a constant level of receptor expression on each cell, a formal reduction may be made such that the models $(18,19)$ and $(21,22)$ have the same homogeneous and inhomogenous steady states. Interestingly, in this limit the models have the same bifurcations even though they are dynamically different due to a multiplicative factor on the right hand side of the bound receptor equation $(21 \mathrm{~b})$.

When binding is not low affinity, our analysis highlights an important difference between square and hexagonal arrays. Although smaller, the pattern region for hexagons with inhibition of ligand production is qualitatively similar to that for a string and squares. The difference is that, in general, stronger ligand inhibition is required for patterns in hexagons when the receptor response is weak or inhibitory, and this condition is also observed in the reduced system. Moreover, when $k_{a}=k_{d} \approx 0$, the condition for patterns in hexagons is exactly 2 times that for strings/squares (i.e. $\mathcal{A}_{-1 / 2}^{*}=2 \mathcal{A}_{-1}^{*}$ ), and this is precisely the relationship found by Collier et al [4]. It is important to note, however, that when binding is not low affinity, the strength of inhibition required for patterns in hexagons $\left(\mathcal{A}_{-1 / 2}^{*}\right)$ is much more negative than that for squares $\left(\mathcal{A}_{-1}^{*}\right)$. For example, with the parameter sets used in this paper, $\mathcal{A}_{-1 / 2}^{*}$ is an order of magnitude larger than $\mathcal{A}_{-1}^{*}$. This means that, unless binding is of low affinity or there is significant activation of receptor production $\left(\mathcal{F}>T_{-1 / 2}\right)$, pattern formation is only favoured in hexagons for unrealistically strong ligand inhibition. It is likely that more realistic cell shapes will be subject to similar constraints as hexagons, and in general a sufficient amount of receptor synthesis will be required for pattern formation. We are currently investigating this feature on discrete irregular geometries.

In addition to the work on irregular networks of cells, there are a number of extensions which could be carried out to the present work. This model and previous work has treated individual cells as single entities, and assumed that all proteins and receptors are equally distributed at the cell surface, and the production terms are similarly homogeneous. We could extend the model to keep track of each membrane surface separately, and thus incorporate biologically relevant features such as localized release of new proteins from intracellular stores, intra-membrane protein transport, and cell polarization. Further complications arise because many membranebound signalling molecules can be cleaved to give a freely diffusible form that can still bind and activate cell surface receptors. Examples include TGF- $\alpha$ and EGF-R in the developing eye of Drosophila [22], and also Delta [33]. It is also of great interest to combine such extracellular diffusion with the juxtacrine signalling framework, and determine its effect on signal propagation and the wavelengths of resulting patterns. Finally, it is important to develop applications to particular developmental systems, and to generate specific testable predictions.

Acknowledgements. SDW was supported by grant no. GR/R46366/01 from the Engineering and Physical Sciences Research Council of Great Britain. We would like to thank the three referees for their helpful comments. 


\section{A. Regions of instability to inhomogeneous perturbations}

Homogeneous steady states of (1) lose stability via a change in sign from positive to negative of $a_{3}$ or $\Delta=a_{1} a_{2}-a_{3}$, which are both quadratic in $K$. Thus, we analyse the critical point, $K_{\mathrm{c}}$, and roots of a quadratic $Q(K)$, given that stability to homogeneous perturbations requires $Q(1)>0$ :

- Region 1: $Q(K)$ has a minimum for some $K_{\mathrm{c}} \in[\kappa, 1)$.

The homogeneous steady state can lose stability via a double root at $K_{\mathrm{c}}: Q\left(K_{\mathrm{c}}\right)=Q^{\prime}\left(K_{\mathrm{c}}\right)=0$.

- Region 2: $Q(K)$ has a maximum, or a minimum for $K_{\mathrm{c}} \notin[\kappa, 1)$.

Bifurcations arise from a single root at $K=\kappa$ given by $Q(\kappa)=0$.

This classification can be found in Table 1, together with sketches of $Q(K)$ illustrating the different bifurcations.

Regions and bifurcations for $a_{3}(K)$.

$$
\begin{aligned}
a_{3}(K) & =-K(\lambda)^{2} k_{a} f_{e}\left(k_{a} a_{e}\left(k_{i}-\mathcal{F}\right)+k_{d} d_{f}\right)-K(\lambda) k_{a} d_{f} f_{e} \mathcal{A} \\
& +\left(k_{a} f_{e}+d_{a}\right)\left(k_{a} a_{e}\left(k_{i}-\mathcal{F}\right)+d_{f}\left(k_{d}+k_{i}\right)\right) .
\end{aligned}
$$

The critical point of $a_{3}(K)$ is at:

$$
K_{\mathrm{c}}=\frac{d f \mathcal{A}}{2 k_{a} a_{e}\left(\mathcal{F}-k_{i}-\frac{k_{d} d_{f}}{k_{a} a_{e}}\right)} .
$$

When $\mathcal{F}>R_{\text {min }}=k_{i}+k_{d} d_{f} / k_{a} a_{e}$, the coefficient of $K^{2}$ is positive, and $K_{\mathrm{c}}$ corresponds to a minimum.

We now find conditions for region 1 - that is, $\mathcal{A}$ and $\mathcal{F}$ such that $K_{\mathrm{c}} \in[\kappa, 1)$. This region is bounded by the lines $R_{\kappa}$ and $R_{1}$ :

$$
\begin{aligned}
R_{-1}: & \mathcal{F}=k_{i}+\frac{2 k_{d} d_{f}-d_{f} \mathcal{A}}{2 k_{a} a_{e}}, \\
R_{-1 / 2}: & \mathcal{F}=k_{i}+\frac{d_{f}\left(k_{d}-\mathcal{A}\right)}{k_{a} a_{e}}, \\
R_{0}: \quad \mathcal{A} & =0, \\
R_{1}: & \mathcal{F}=k_{i}+\frac{2 k_{d} d_{f}+d_{f} \mathcal{A}}{2 k_{a} a_{e}} .
\end{aligned}
$$

These lines all intersect at $\left(\mathcal{A}=0, \mathcal{F}=R_{\text {min }}\right)$, and combined with the condition for a minimum, they subdivide the $(\mathcal{A}, \mathcal{F})$ plane into two regions (see Figure $3(\mathrm{a})$ ). Region 1 is given by $\mathcal{F}>\max \left\{R_{\kappa}, R_{1}\right\}$. 
The bifurcations $a_{3}(\kappa)=0$ and $a_{3}\left(K_{\mathrm{c}}\right)=0$ occur on $T_{\kappa}$ and $T_{d}$, respectively, where

$$
\begin{aligned}
T_{-1}: & \mathcal{F}=k_{i}+\frac{d_{f}\left(k_{d}+k_{i}\right)}{k_{a} a_{e}}+\frac{d_{f} f_{e}\left(k_{i}+\mathcal{A}\right)}{d_{a} a_{e}} \\
T_{-1 / 2}: & \mathcal{F}=k_{i}+\frac{4 d_{a} d_{f}\left(k_{d}+k_{i}\right)}{k_{a} a_{e}\left(3 k_{a} f_{e}+4 d_{a}\right)}+\frac{d_{f} f_{e}\left(3 k_{d}+4 k_{i}+2 \mathcal{A}\right)}{a_{e}\left(3 k_{a} f_{e}+4 d_{a}\right)} \\
T_{0}: & \mathcal{F}=k_{i}+\frac{d_{f}\left(k_{d}+k_{i}\right)}{k_{a} a_{e}} \\
T_{d}^{ \pm}: & \mathcal{F}=k_{i}+\frac{d_{f}}{2 k_{a} a_{e}}\left(2 k_{d}+k_{i} \pm \sqrt{k_{i}^{2}-\frac{k_{a} f_{e} \mathcal{A}^{2}}{k_{a} f_{e}+d_{a}}}\right)
\end{aligned}
$$

See Figure 3(b) for a qualitative illustration of these curves in the $(\mathcal{A}, \mathcal{F})$ plane. In summary:

- Region 1: $a_{3}(K)$ has a minimum at $K_{\mathrm{c}} \in[\kappa, 1)$ when $\mathcal{F}>\max \left\{R_{\kappa}, R_{1}\right\}$. $a_{3}\left(K_{c}\right)<0$ for $\mathcal{F}>T_{d}^{+}$.

- Region 2: $a_{3}(K)$ does not have a minimum in $[\kappa, 1)$ for $\mathcal{F}<\max \left\{R_{\kappa}, R_{1}\right\}$. $a_{3}(\kappa)<0$ for $\mathcal{F}>T_{\kappa}$.

Regions and bifurcations for $\Delta(K)$.

$$
\begin{aligned}
\Delta(K)= & -k_{a} f_{e}\left(k_{a} a_{e}\left(a_{1}+\mathcal{F}-k_{i}\right)+k_{d}\left(a_{1}-d_{f}\right)\right) K^{2} \\
& -k_{a} f_{e} \mathcal{A}\left(a_{1}-d_{f}\right) K \\
& +\left(k_{a} a_{e}+d_{f}+k_{d}+k_{i}\right)\left(k_{a} f_{e}\left(a_{1}+d_{a}\right)+k_{a} a_{e}\left(d_{a}+k_{i}-\mathcal{F}\right)\right. \\
& \left.+\left(d_{a}+d_{f}\right)\left(d_{a}+k_{i}+k_{d}\right)\right)
\end{aligned}
$$

The critical point for $\Delta(K)$ is at:

$$
K_{\mathrm{c}}=\frac{-\mathcal{A}\left(a_{1}-d_{f}\right)}{2\left(k_{a} a_{e}\left(a_{1}+\mathcal{F}-k_{i}\right)+k_{d}\left(a_{1}-d_{f}\right)\right)} .
$$


When $\mathcal{F}<S_{\text {min }}=k_{i}-a_{1}-\frac{k_{d}\left(a_{1}-d_{f}\right)}{k_{a} a_{e}}, K_{\mathrm{c}}$ corresponds to a minimum. Solving $K_{\mathrm{c}}=-1,-1 / 2,0$ and 1 yields

$$
\begin{aligned}
S_{-1}: & \mathcal{F}=k_{i}-a_{1}+\frac{\left(\mathcal{A}-2 k_{d}\right)\left(a_{1}-d_{f}\right)}{2 k_{a} a_{e}}, \\
S_{-1 / 2}: & \mathcal{F}=k_{i}-a_{1}+\frac{\left(\mathcal{A}-k_{d}\right)\left(a_{1}-d_{f}\right)}{k_{a} a_{e}}, \\
S_{0}: \quad \mathcal{A} & =0, \\
S_{1}: & \mathcal{F}=k_{i}-a_{1}-\frac{\left(\mathcal{A}+2 k_{d}\right)\left(a_{1}-d_{f}\right)}{2 k_{a} a_{e}} .
\end{aligned}
$$

These lines all intersect at $\left(\mathcal{A}=0, \mathcal{F}=S_{\text {min }}\right)$, as illustrated in Figure 4(a). With the above condition for a minimum, region 1 for $Q(K)=\Delta(K)$ is given by $\mathcal{F}<\min \left\{S_{\kappa}, S_{1}\right\}$.

The bifurcations $\Delta(\kappa)=0$ and $\Delta\left(K_{\mathrm{c}}\right)=0$ occur on the curves $H_{\kappa}$ and $H_{d}$, respectively:

$$
\begin{aligned}
H_{-1}: \quad \mathcal{F} & =k_{i}+d_{a}+\frac{d_{f} f_{e}}{a_{e}}+\frac{d_{a} d_{f}+\left(d_{a}+d_{f}\right)\left(k_{d}+k_{i}\right)}{k_{a} a_{e}} \\
& +\frac{d_{a}^{2}\left(d_{f}+k_{d}+k_{i}\right)+d_{a} k_{a}\left(d_{f} f_{e}+d_{a} a_{e}\right)+k_{a} k_{i} f_{e}\left(a 1-d_{f}\right)}{k_{a} a_{e}\left(a_{1}-d_{a}\right)} \\
& +\frac{f_{e}\left(a_{1}-d_{f}\right) \mathcal{A}}{a_{e}\left(a_{1}-d_{a}\right)}, \\
H_{-1 / 2}: \quad \mathcal{F} & =k_{i}+d_{a}+\frac{3 k_{a} f_{e}\left(a_{1}+d_{a}\right)}{4 a_{1}-4 d_{a}-3 k_{a} f_{e}}+\frac{3 f_{e} k_{d}\left(a_{1}-d_{f}\right)+4 f_{e} d_{f}\left(a_{1}-k_{i}\right)}{a_{e}\left(4 a_{1}-4 d_{a}-3 k_{a} f_{e}\right.} \\
& +\frac{\left(4\left(d_{a}+d_{f}\right) a_{1}-4 d_{a} d_{f}\right)\left(k_{d}+k_{i}\right)+4 k_{a} a_{e} d_{a}^{2}+4 a_{1}\left(d_{a} d_{f}+k_{a} f_{e} k_{i}\right)}{k_{a} a_{e}\left(4 a_{1}-4 d_{a}-3 k_{a} f_{e}\right)} \\
H_{0}: \quad & \mathcal{F}=d_{a}+k_{i}+\frac{f_{e}\left(a_{1}+d_{a}\right)}{a_{e}}+\frac{\left(d_{a}+d_{f}\right)\left(d_{a}+k_{d}+k_{i}\right)}{k_{a} a_{e}}, \\
& +\frac{2 f_{e}\left(a_{1}-d_{f}\right) \mathcal{A}}{a_{e}\left(4 a_{1}-4 d_{a}-3 k_{a} f_{e}\right)},
\end{aligned}
$$




$$
H_{d}: \mathcal{F}=\frac{-\delta_{1}}{2 \delta_{2}} \pm \frac{1}{2 \delta_{2}} \sqrt{\delta_{1}^{2}-4 \delta_{0} \delta_{2}}
$$

where

$$
\begin{aligned}
\delta_{2}= & -4 k_{a}^{2} a_{e}^{2}\left(k_{a} a_{e}+d_{f}+k_{d}+k_{i}\right), \\
\delta_{1}= & 4 k_{a} a_{e}\left(k_{a} a_{e}+d_{f}+k_{d}+k_{i}\right)\left(k_{a} f_{e}\left(a_{1}+d_{a}\right)+k_{a} a_{e}\left(d_{a}+2 k_{i}-a_{1}\right)\right. \\
& \left.\quad-k_{d}\left(a_{1}-d_{f}\right)+\left(d_{a}+d_{f}\right)\left(d_{a}+k_{i}+k_{d}\right)\right), \\
\delta_{0}= & k_{a} f_{e} \mathcal{A}^{2}\left(a_{1}-d_{f}\right)^{2}+4\left(k_{a} a_{e}\left(a_{1}-k_{i}\right)+k_{d}\left(a_{1}-d_{f}\right)\right)\left(k_{a} a_{e}+d_{f}+k_{d}+k_{i}\right) \\
& \times\left(k_{a} f_{e}\left(a_{1}+d_{a}\right)+k_{a} a_{e}\left(d_{a}+k_{i}\right)+\left(d_{a}+d_{f}\right)\left(d_{a}+k_{i}+k_{d}\right)\right) .
\end{aligned}
$$

See Figure 4(b) for a qualitative illustration of these curves. To summarise:

- Region 1: $\Delta(K)$ has a minimum at $K_{\mathrm{c}} \in[\kappa, 1)$ when $\mathcal{F}<\min \left\{S_{\kappa}, S_{1}\right\}$. $\Delta\left(K_{c}\right)<0$ for $H_{d}^{-}<\mathcal{F}<H_{d}^{+}$.

- Region 2: $\Delta(K)$ does not have a minimum in $[\kappa, 1)$ for $\mathcal{F}>\min \left\{S_{\kappa}, S_{1}\right\}$. $\Delta(\kappa)<0$ for $\mathcal{F}>H_{\kappa}$.

\section{B. Parameter values}

In our numerical simulations, we use parameter values based on experimental data for binding of epidermal growth factor to its receptors. Unless otherwise stated, the rate constants are taken as: $k_{a}=0.0003 \mathrm{molecule}^{-1} \mathrm{~min}^{-1}$, $k_{d}=0.12 \mathrm{~min}^{-1}, k_{i}=0.019 \mathrm{~min}^{-1}, d_{a}=0.01 \mathrm{~min}^{-1}$, and $d_{f}=0.03 \mathrm{~min}^{-1}$. An explanation of the choice of each individual value can be found in the work of Owen \& Sherratt [28]. The same values were also used by Wearing et al [40].

The production functions are of Hill form such that

$$
P_{a}(b)=\frac{C_{1} b^{m}}{C_{2}^{m}+b^{m}}, \quad \text { and } \quad P_{f}(b)=C_{3}+\frac{C_{4} b^{n}}{C_{5}^{n}+b^{n}} .
$$

The Hill function is a commonly used example for nonlinear responses in this type of system. Note that inhibition is given by choosing negative exponents - this is equivalent to the more usual decreasing Hill function form $P_{a}(b)=$ $C_{1} C_{2}^{h} /\left(C_{2}^{h}+b^{h}\right)$ where $h=-m$, and similarly for $P_{f}(b)$.

We investigate a particular case in which the parameter values are fixed, except for two free parameters that can be interpreted as a measure of the response strengths of ligand and receptor production. Experimentally testable quantities specify some of the parameters. For example, in the absence of ligand there will be some background level of receptor expression. This is one of the homogeneous steady states of the model, and so (1b) gives

$$
P_{f}(0)=d_{f} r_{0},
$$


where $r_{0}$ is the unstimulated receptor number. Furthermore, if we fix the normal equilibrium levels of free and bound receptors, say $f_{e}$ and $b_{e}$, then (1c) defines the normal steady state level of free ligand, $a_{e}$, as well as the values of the production functions at that steady state, so that

$$
a_{e}=\frac{\left(k_{d}+k_{i}\right) b_{e}}{k_{a} f_{e}}, \quad P_{a}\left(b_{e}\right)=k_{i} b_{e}+d_{a} a_{e} \quad \text { and } \quad P_{f}\left(b_{e}\right)=k_{i} b_{e}+d_{f} f_{e} .
$$

These conditions fix the parameters $C_{1}, C_{3}$ and $C_{4}$, to give

$$
C_{1}=\left(k_{i} b_{e}+d_{a} a_{e}\right) \frac{\left(C_{2}^{m}+b_{e}^{m}\right)}{b_{e}^{m}}, \quad C_{3}=d_{f} r_{0},
$$

and

$$
C_{4}=\left(k_{i} b_{e}+d_{f}\left(f_{e}-r_{0}\right)\right) \frac{\left(C_{2}^{m}+b_{e}^{m}\right)}{b_{e}^{m}} .
$$

In the linear analysis the key regulators of the model behaviour are the slopes of the production functions at the homogeneous steady state. Writing $C_{2}=\beta_{a} b_{e}$ and $C_{5}=\beta_{f} b_{e}$, gives

$\mathcal{A} \equiv P_{a}^{\prime}\left(b_{e}\right)=\frac{m\left(k_{i} b_{e}+d_{a} a_{e}\right) \beta_{a}^{m}}{b_{e}\left(\beta_{a}^{m}+1\right)}, \quad \mathcal{F} \equiv P_{f}^{\prime}\left(b_{e}\right)=\frac{n\left(k_{i} b_{e}+d_{f}\left(f_{e}-r_{0}\right)\right) \beta_{f}^{n}}{b_{e}\left(\beta_{f}^{n}+1\right)}$.

If $\beta_{a}=\beta_{f}=1, \mathcal{A}$ and $\mathcal{F}$ are linear functions of $m$ and $n$, so that we can use $m, n$ to fix $\mathcal{A}, \mathcal{F} \in(-\infty, \infty)$. If $\beta_{a}, \beta_{f} \neq 1$, varying $m$ and $n$ does not give entire ranges for $\mathcal{A}$ and $\mathcal{F}$. For example, when $\beta_{a}<1, \mathcal{A}$ is strictly increasing for $m<0$, has a maximum for some $m>0$, and then tends to zero as $m$ gets large. For $\beta_{a}>1, \mathcal{A}$ is strictly increasing for $m>0$, and has a turning point for some $m<0$. The same features are seen for $\mathcal{F}$ as $\beta_{f}$ varies through 1 . Unless otherwise stated, we take $\beta_{a}=\beta_{f}=1$, as well as $r_{0}=f_{e}=b_{e}=3000$ molecules per cell.

\section{References}

1. R E Brandt and L E Vickery. Cooperativity and dimerization of recombinant human estrogen receptor hormone-binding domain. J. Biol. Chem., 272:48434849, 1997.

2. A J L Clark, S Ishii, N Richert, G T Merlino, and I Pastan. Epidermal growth factor regulates the expression of its own receptor. Proc. Nat. Acad. Sci. USA, 82:8374-8378, 1985.

3. R J Coffey, R Derynck, J N Wilcox, T S Bringman, A S Goustin, H L Moses, and M R Pittelkow. Production and auto-induction of transforming growth Factor- $\alpha$ in human keratinocytes. Nature, 328:817-820, 1987.

4. J R Collier, N A M Monk, P K Maini, and J H Lewis. Pattern formation by lateral inhibition with feedback: A mathematical model of delta-notch intercellular signalling. J. Theor. Biol., 183:429-446, 1996.

5. J F de Celis and S Bray. Feedback mechanisms affecting notch activation at the dorsoventral boundary in the Drosophila wing. Development, 124:3241$3251,1997$. 
6. E J Doedel, A R Champneys, $T \mathrm{R}$ Fairgrieve, $\mathrm{Y}$ A Kuznetsov, B Sandstede, and X J Wang. AUTO 97: Continuation and bifurcation software for ordinary differential equations (1997). Available from http://indy.cs.concordia.ca/auto/main.html.

7. F Fagotto and B M Gumbiner. Cell contact dependent signalling. Dev. Biol., 180:445-454, 1996.

8. B Fanger, J E Stephens, and J V Staros. High yield trapping of EGF-induced receptor dimers by cross-linking. FASEB, 3:71-75, 1989.

9. L J Fretto, A J Snape J E Tomlinson, J J Seroogy, D L Wolf, W J LaRochelle, and N A Giese. Mechanism of platelet-derived growth-factor (PDGF)-AA, (PDGF)-AB, and (PDGF)-BB binding to alpha-PDGF and beta -PDGF receptor. J. Biol. Chem., 268:3625-3631, 1993.

10. H Hirata, S Yoshiura, T Ohtsuka, Y Bessho, T Harada, K Yoshikawa, and $\mathrm{R}$ Kageyama. Oscillatory expression of the bHLH factor Hes1 regulated by a negative feedback loop. Science, 298:840-843, 2002.

11. A D Hughes, G F Clunn, J Refson, and C Demoliou-Mason. Platelet-derived growth factor (PDGF): Actions and mechanisms in vascular smooth muscle. Gen. Pharmac., 27:1079-1089, 1996.

12. S S Huppert, T L Jacobson, and M A T Muskavitch. Feedback regulation is central to Delta-Notch signalling required for Drosophila wing vein morphogenesis. Development, 124:3283-3291, 1997.

13. Y Jiang, B L Aerne, L Smithers, C Haddon, D Ish-Horowicz, and J Lewis. Notch signalling and the synchronization of the somite segmentation clock. Nature, 408:475-479, 2000.

14. C Jouve, I Palmeirim, D Henrique, J Beckers, A Gossler, D Ish-Horowicz, and O Pourquié. Notch signalling is required for cyclic expression of the hairy-like gene HES1 in the presomitic mesoderm. Development, 127:1421-1429, 2000.

15. M Kerszberg and L Wolpert. Mechanisms for positional signalling by morphogen transport: a theoretical study. J. Theor. Biol., 191:103-114, 1998.

16. J Kimble and $\mathrm{P}$ Simpson. The LIN-12/Notch signalling pathway and its regulation. Ann. Rev. Cell Dev. Biol., 13:333-361, 1997.

17. K M Klueg, T R Parody, and M A Muskavitch. Complex proteolytic processing acts on Delta, a transmembrane ligand for Notch, during Drosophila development. Mol. Biol. Cell, 9:1709-1723, 1998.

18. A D Lander, Q Nie, and F W M Wan. Do morphogen gradients arise by diffusion. Dev. Cell., 2:785-796, 2002.

19. M A Lemmon, D Pinchasi, M Zhou, I lax, and J Schlessinger. Kit receptor dimerization is driven by bivalent binding of stem cell factor. J. Biol. Chem., 272:6311-6317, 1997.

20. J Lewis. Neurogenic genes and vertebrate neurogenesis. Curr. Op. Neurobiol., 6:3-10, 1996.

21. J Lewis. Notch signalling and the control of cell fate choices in vertebrates. Semin. Cell Dev. Biol., 9:583-589, 1998.

22. J Massagué and A Pandiella. Membrane-anchored growth factors. Annu. Rev. Biochem., 62:515-541, 1993.

23. H Meinhardt. Models of biological pattern formation. Academic Press, London, 1982.

24. E Meir, G von Dassow, E Munro, and G M Odell. Robustness, flexibility and the role of lateral inhibition in the neurogenic network. Curr. Biol., 12:778-786, 2002.

25. N A M Monk. Restricted-range gradients and travelling fronts in a model of juxtacrine cell relay. Bull. Math. Biol., 60:901-918, 1998.

26. J D Murray. Mathematical Biology. Springer Verlag, Berlin, 1989.

27. M R Owen. Waves and propagation failure in discrete space models with nonlinear coupling and feedback. Physica D, 173:59-76, 2002. 
28. M R Owen and J A Sherratt. Mathematical modelling of juxtacrine cell signalling. Math. Biosci., 152:125-150, 1998.

29. M R Owen, J A Sherratt, and H J Wearing. Lateral induction by juxtacrine signalling is a new mechanism for pattern formation. Dev. Biol., 217:54-61, 2000 .

30. K J Painter, P K Maini, and H G Othmer. Stripe formation in juvenile Pomecanthus explained by a generalized Turing mechanism with chemotaxis. Proc. Nat. Acad. Sci. USA, 96:5549-5554, 1999.

31. V M Panin, V Papayannopoulos, R Wilson, and K D Irvine. Fringe modulates Notch-ligand interactions. Nature, 387:908-912, 1997.

32. E Plahte. Pattern formation in discrete cell lattices. J. Math. Biol., 43:411445, 2001.

33. H Qi, M D Rand, X Wu, N Sestan, W Wang, P Rakic, T Xu, and S ArtavanisTsakonas. Processing of the Notch ligand Delta by the metalloprotease Kuzbanian. Science, 283:91-94, 1999.

34. K M Reilly and D A Melton. Short-range signalling by candidate morphogens of the TGF beta family and evidence for a relay mechanism of induction. Cell, 86:743-754, 1996.

35. S Schnell and P K Maini. Clock and induction model for somitogenesis. Dev. Dyn., 217:415-420, 2000.

36. J B Skeath and S B Carroll. Regulation of proneural gene expression and cell fate during neuroblast segregation in the drosophila embryo. Development, 114:939-946, 1992.

37. T Spivak-Kroizman, M A Lemmon, I Dikic, J E Ladbury, D Pinchasi, J Huang, M Jaye, G Crumley, G Schlessinger, and I Lax. Heparin-induced oligomerization of FGF molecules is responsible for FGF receptor dimerization, activation, and cell proliferation. Cell, 79:1015-1024, 1994.

38. A M Turing. The chemical basis of morphogenesis. Phil. Trans. R. Soc. Lond. $B, 237: 37-72,1952$.

39. C M Waters, K C Oberg, G Carpenter, and K A Overholser. Rate constants for binding, dissociation, and internalization of EGF: Effect of receptor occupancy and ligand concentration. Biochemistry, 29:3563-3569, 1990.

40. H J Wearing, M R Owen, and J A Sherratt. Mathematical modelling of juxtacrine patterning. Bull. Math. Biol., 62:293-320, 2000.

41. H J Wearing and J A Sherratt. Nonlinear analysis of juxtacrine patterns. SIAM J. Appl. Math., 62:283-309, 2001.

42. T Whitfield, C Haddon, and J Lewis. Intercellular signals and cell-fate choices in the developing inner ear: origins of global and of fine-grained pattern. Sem. Cell Dev. Biol., 8:239-247, 1997.

43. L Wolpert. Positional information and the spatial pattern of cellular differentiation. J. Theor. Biol., 25:1-47, 1969. 


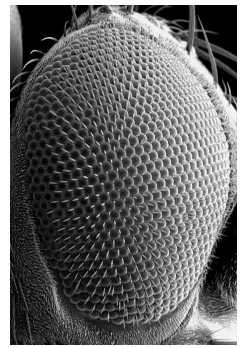

(a)

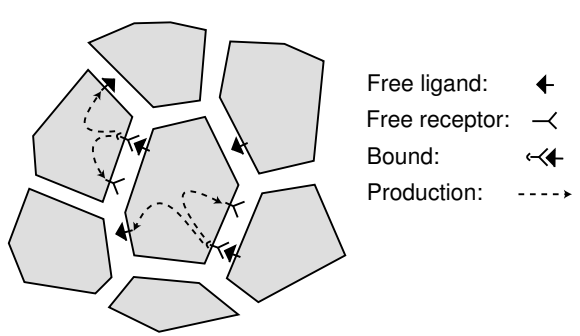

(b)

Fig. 1. (a) Patterns in the eye of the fruit fly Drosophila. The fly's eye is composed of a reiterated pattern of about 750 unit eyes known as ommatidia. Each ommatidia has the same internal structure being composed of eight photoreceptor neurons or retinula cells (Image courtesy of Kevin Moses, http://www .emory. edu/CELLBIO/moses/). (b) Juxtacrine signalling: ligands on the surface of one cell bind to free receptors on a neighbouring surface, governing production of new ligand and receptors.
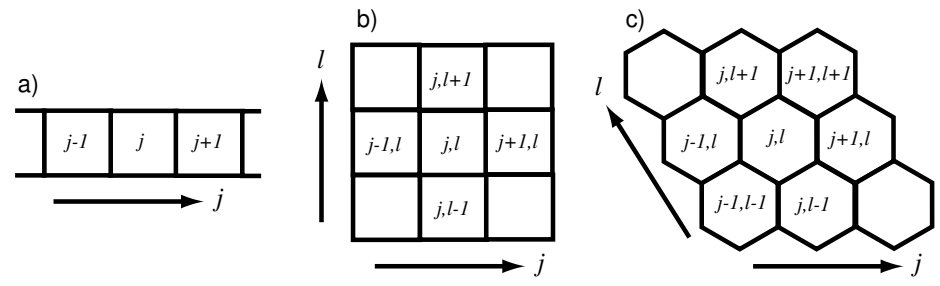

Fig. 2. We study the juxtacrine signalling model (1) on a one-dimensional linear array and two-dimensional cellular arrays of squares and hexagons. Here we indicate the spatial labelling scheme. 
(a)

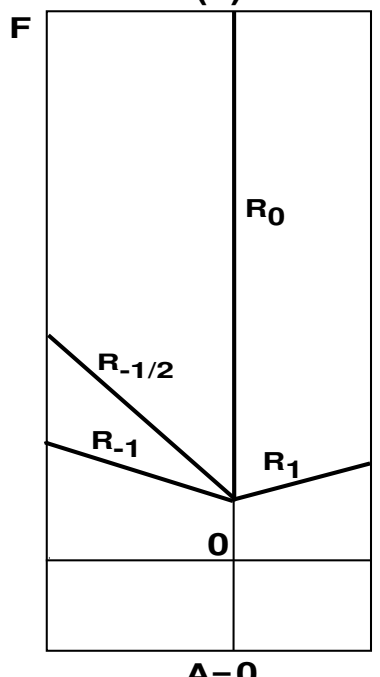

(b)

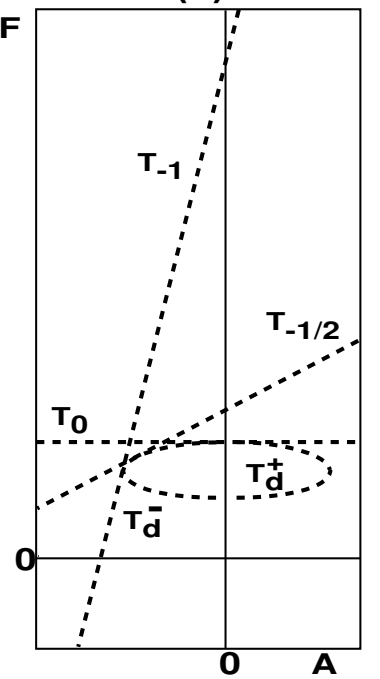

(c)

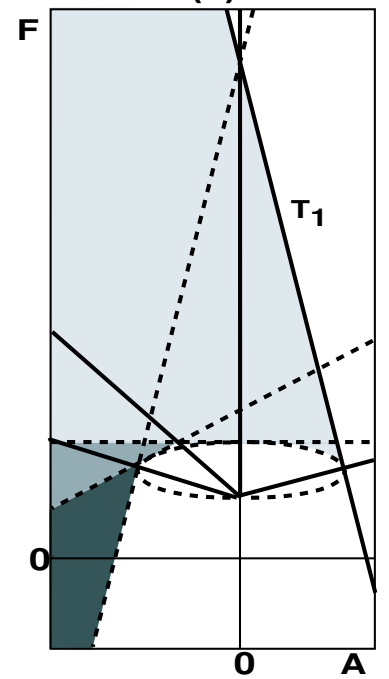

Fig. 3. Curves in $(\mathcal{A}, \mathcal{F})$ space which delimit the regions where $a_{3}(1)>0$ (for stability to homogeneous perturbations) and $a_{3}(K)<0$ for some $K \in[\kappa, 1$ ) (for spatial instability), where $\kappa=-1$ for strings/squares, $\kappa=-1 / 2$ for hexagons, and $\kappa=0$ for striped patterns in square arrays. (a) $a_{3}(K)$ has a minimum at $K_{\mathrm{c}} \in[\kappa, 1)$ when $\mathcal{F}>\max \left\{R_{\kappa}, R_{1}\right\}$. This allows for the possibility of patterning bifurcation via a double root $a_{3}\left(K_{c}\right)=0$, on the curve $T_{d}^{ \pm}$shown in part (b). Alternatively, a patterning bifurcation can occur via a single root $a_{3}(\kappa)=0$, along the line $T_{\kappa}$. In (c), the lightest shading is where $a_{3}(K)<0$ for some $K \in[0,1)$, which gives unstable modes for all the geometries considered here. The intermediate shading is where $a_{3}(K)<0$ for some $K \in[-1 / 2,0)$, giving unstable modes only for hexagons and strings/squares. In the darkest region $a_{3}(K)<0$ for some $K \in[-1,-1 / 2)$, which only gives patterns for strings/squares. 
(a)

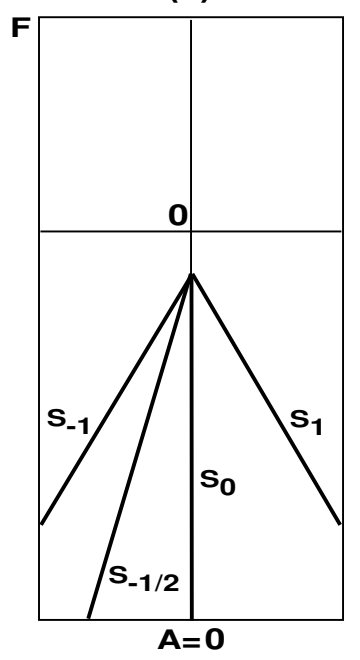

(b)

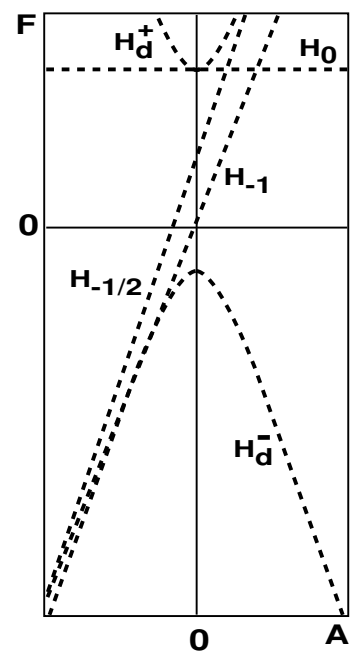

(c)

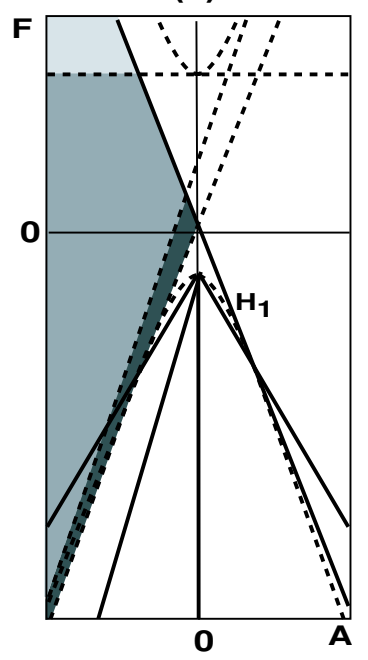

Fig. 4. Curves in $(\mathcal{A}, \mathcal{F})$ space which delimit the regions where $\Delta(1)>0$ (for stability to homogeneous perturbations) and $\Delta(K)<0$ for some $K \in[\kappa, 1$ ) (for an oscillatory spatial instability), where $\kappa=-1$ for strings/squares, $\kappa=-1 / 2$ for hexagons, and $\kappa=0$ for striped patterns in square arrays. The details are as in Figure 3, replacing $a_{3}$ with $\Delta, R$ with $S$, and $T$ with $H$. 
(a)

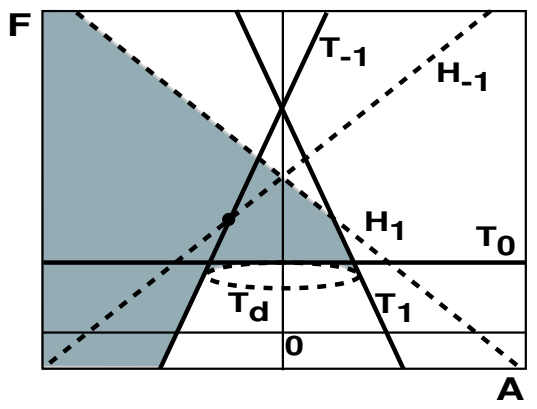

(b)
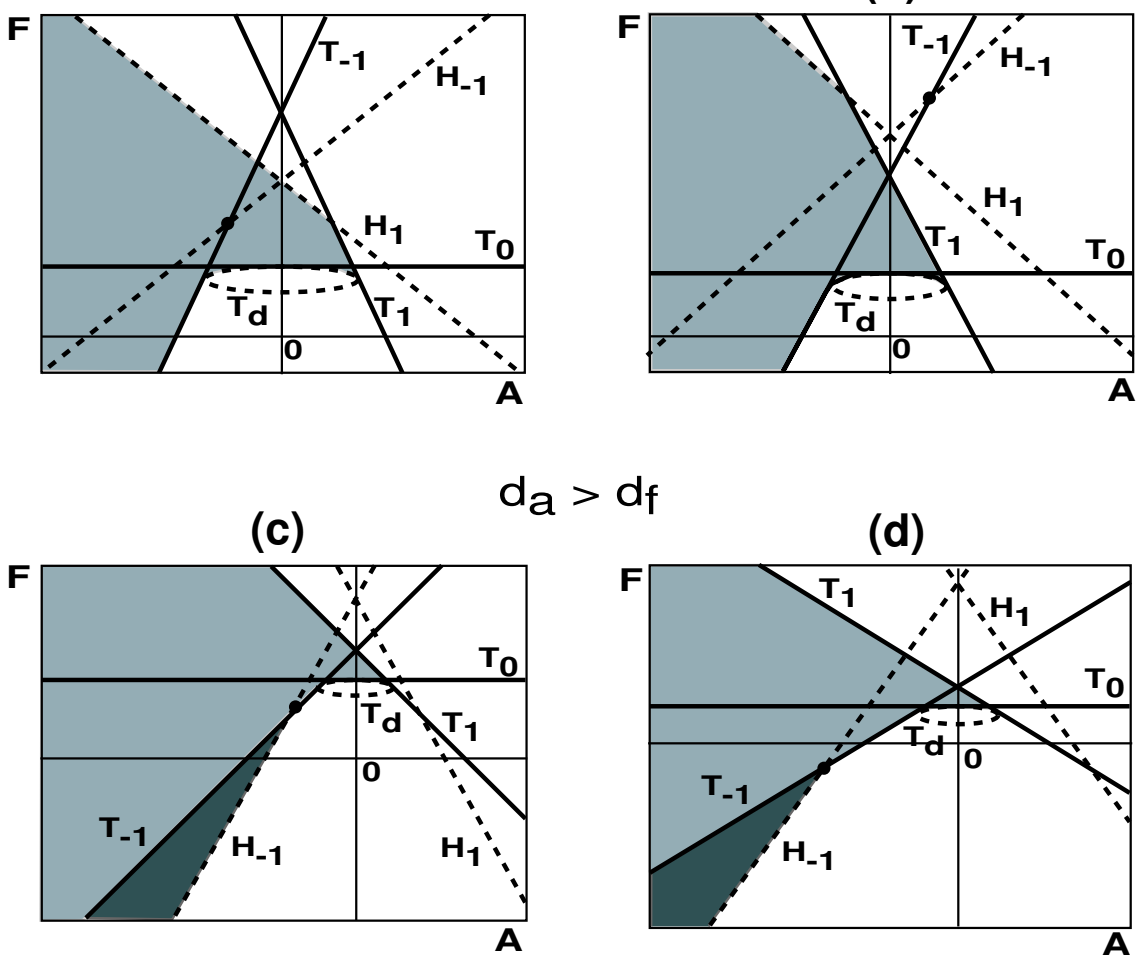

(d)

$\mathrm{d}_{\mathrm{a}}>\mathrm{d}_{\mathrm{f}}$

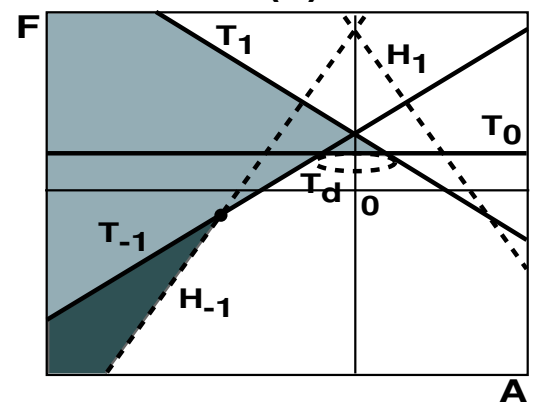

Fig. 5. Qualitative illustration of the regions in the $(\mathcal{A}, \mathcal{F})$ parameter space where pattern formation is predicted for strings/squares. The homogeneous steady state is stable to homogeneous perturbations when $\mathcal{F}<\min \left\{T_{1}, H_{1}\right\}$, and unstable to inhomogeneous perturbations for $\mathcal{F}>\min \left\{T_{d}^{+}, T_{-1}, H_{d}^{-}, H_{-1}\right\}$. This is the union of the shaded regions in parts (c) of Figures 3 and 4 below $T_{1}$ and $H_{1}$. In (a) and (b), $d_{f}>d_{a}$, so that $T_{-1}$ has steeper slope than $H_{-1}$, and their intersection must be at $\mathcal{F}>T_{0}$. Cases (c) and (d) correspond to $d_{f}<d_{a}$ : here $H_{-1}$ is steeper than $T_{-1}$ and the instability can occur via a Hopf bifurcation along the line $H_{-1}$. The dark shading indicates the region where linear stability analysis predicts oscillatory patterns. The picture for hexagons is similar except that the relative slopes of the two lines $T_{-1 / 2}$ and $H_{-1 / 2}$ depend on $k_{a}$ and $f_{e}$ as well as $d_{a}$ and $d_{f}$. 
(a)

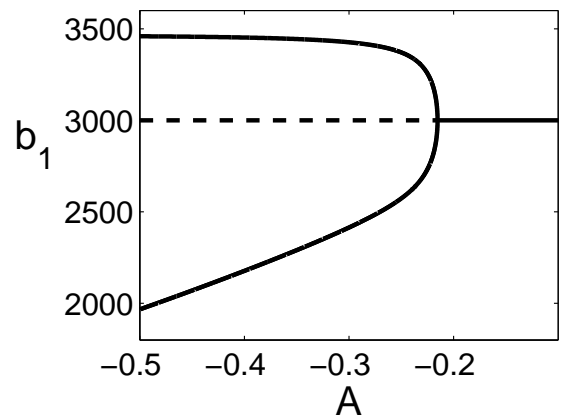

(b)

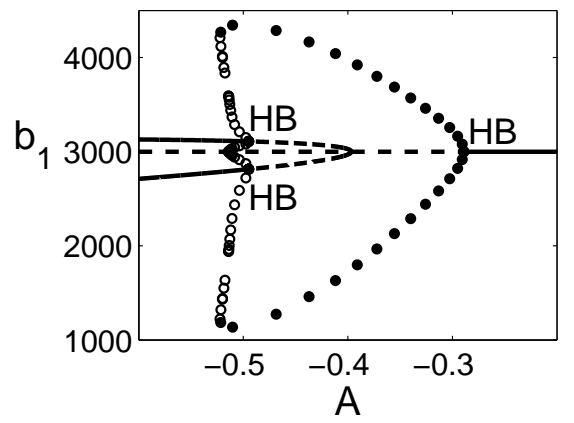

Fig. 6. Bifurcation diagrams for the juxtacrine signalling model (1), with two cells and periodic boundary conditions, produced with AUTO [6]. We vary $\mathcal{A}$ along the line $\mathcal{F}=0$ in parts (a) and (c) of Figure 5, and we plot the bound receptor variable for one cell. Stable (unstable) stationary solutions are solid (dashed), and solid (open) circles indicate stable (unstable) limit cycle oscillations. In part (a), as $\mathcal{A}$ decreases, the homogeneous steady state loses stability at $\mathcal{A}=-0.215$, in favour of a pair of non-uniform states $\left(b_{1}, b_{2}\right)$ and $\left(b_{2}, b_{1}\right)$. In part (b), when $\mathcal{A}=-0.289, \Delta(K)=0$ and $a_{3}(K)>0$, so that there is a Hopf bifurcationthe levels of molecules on the two cells oscillate out of phase. As $\mathcal{A}$ is decreased further a limit point is reached, at which periodic solutions are lost in favour of a stable heterogeneous steady state. Prior to this limit point, there is an interval of $\mathcal{A}$ where both these types of solution coexist - this behaviour arises from a subcritical Hopf bifurcation of the steady heterogeneous solution at $\mathcal{A}=-0.495$. Parameters are given in Appendix B, except for $k_{i}=0.19 \mathrm{~min}^{-1}$, and in part (b) $d_{a}=0.03 \mathrm{~min}^{-1}, d_{f}=0.01 \mathrm{~min}^{-1}$. 
(a) Stationary

2500 mon

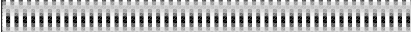
2000 r min:

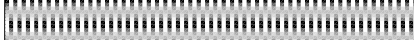

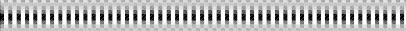

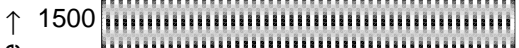

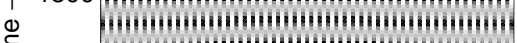

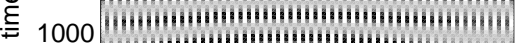

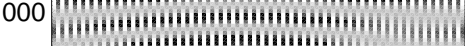

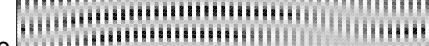

500 rinch

7im

$\begin{array}{lllll}0 & 0 & 40 \quad 60 & 80 & 100\end{array}$ cell number (b) Travelling

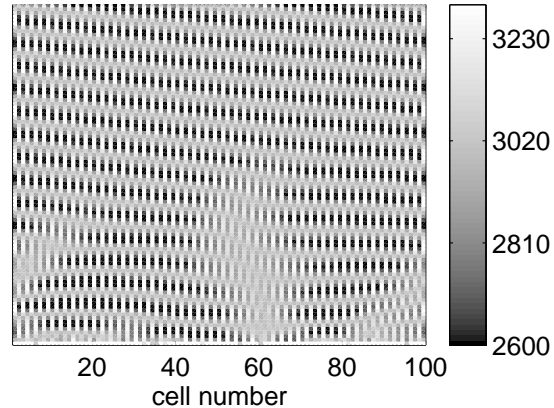

Fig. 7. Standing waves and travelling waves in the juxtacrine signalling model (1), solved on a string of 100 cells with periodic boundary conditions. We plot the densities of bound receptors at successive times from $t=0$ to $t=2500$. Ligand and free receptor have a similar profile. The initial conditions are small random perturbations about the homogeneous steady state. The ligand and receptor production parameters are $m=-2.8$ and $n=1$; other parameter values are given in Appendix B, except for $k_{i}=0.19 \mathrm{~min}^{-1}, d_{a}=0.03 \mathrm{~min}^{-1}, d_{f}=0.01 \mathrm{~min}^{-1}$ - these values give $\mathcal{A}=-0.281$ and $\mathcal{F}=0.095$. Linear analysis predicts the formation of oscillating patterns. In (a), the system evolves to a standing wave. Different random initial conditions gives rise to a travelling wave in (b). 
(a)

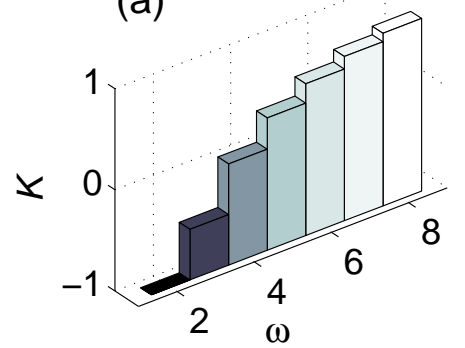

(c)

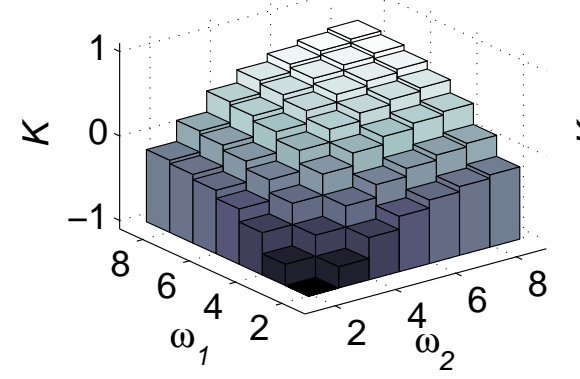

(b)

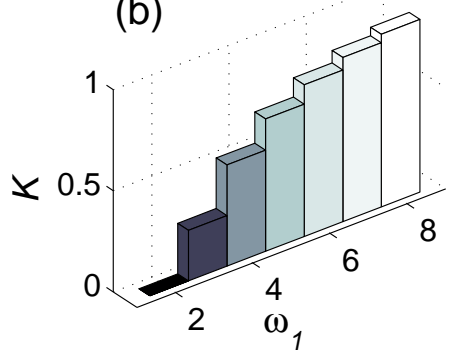

(d)

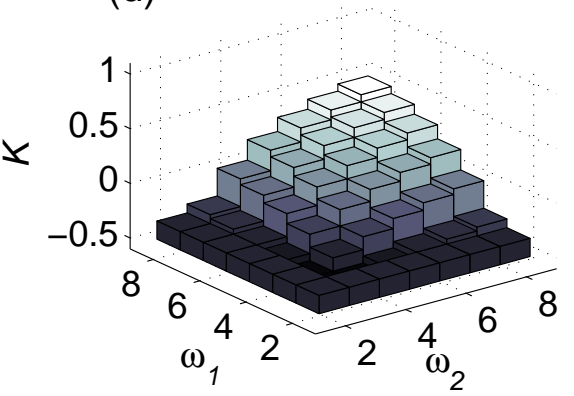

Fig. 8. The relationship between $K$ and the integer wavelengths of regular patterns in discrete cellular arrays, defined in (11): (a) string; (b) for stripes in square arrays; (c) squares; (d) hexagons. In (a) and (b), regular patterns are characterised by a single integer wavelength $\omega$, giving a unique value of $K$. In (c) and (d), twodimensional patterns are specified by two integer wavelengths, $\omega_{1}$ and $\omega_{2}$, and there is a much finer gradation of $K$. In addition, different combinations of $\omega_{1}$ and $\omega_{2}$ give the same value of $K$, so that more than one different pattern can have the same linear growth rate. For squares (c), the minimum value of $K$ is -1 , corresponding to a wavelength of 2 cells, i.e. $\omega_{1}=\omega_{2}=2$. For hexagons, the minimum value is $K=-1 / 2$, corresponding a wavelength $3 \times 3$. 
(a) String

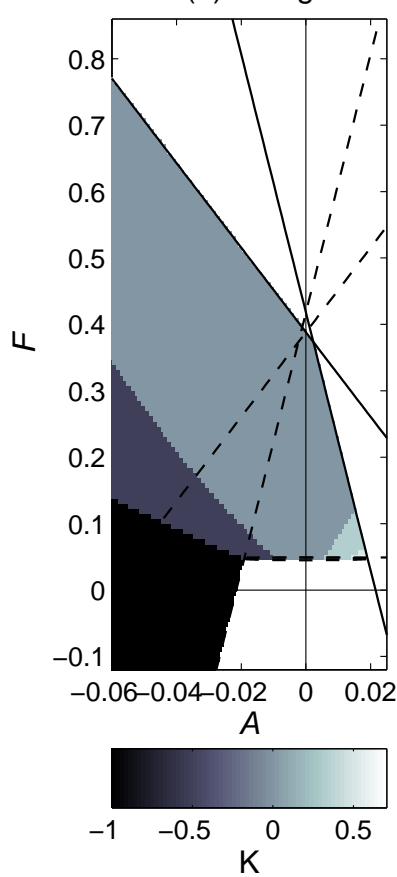

(b) Squares
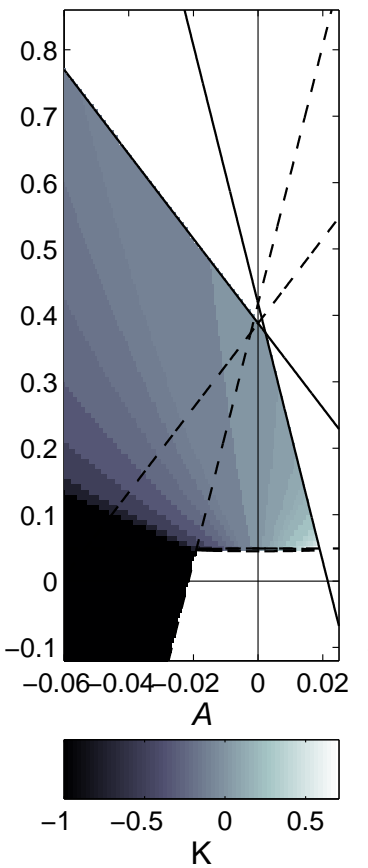

(c) Hexagons

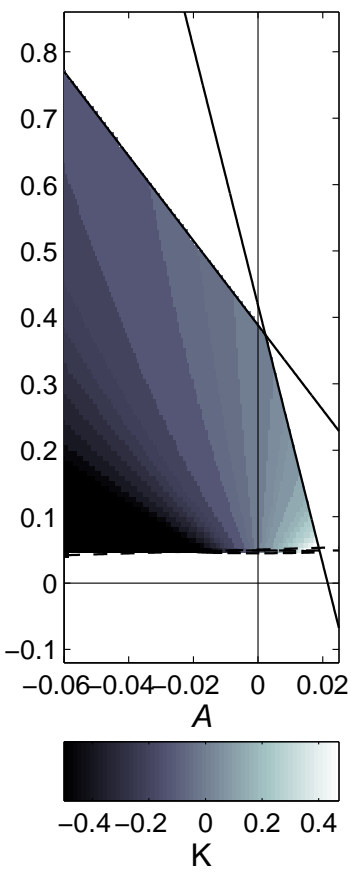

Fig. 9. An example of the variation with $\mathcal{A}$ and $\mathcal{F}$ ) of the fastest growing mode $K$, corresponding to regular patterns with integer wavelengths. Areas that are not shaded are either unstable to homogeneous perturbations or stable to inhomogeneous perturbations. The grey scale used is the same as in Figure 8, which establishes the relationship between the value of $K$ and the wavelengths of regular patterns. For example, for a string in (a), black is $K=-1$, giving a fastest growing integer wavelength of 2 cells; intermediate shading is $K=0$, wavelength 4 ; and light grey is where $K$ is close to 1 indicating longer wavelengths $(>8)$. For hexagons in (c) the minimum value of $K$ is $-1 / 2$, so that black shading corresponds to wavelength $3 \times 3$. The parameter values are as in Appendix B, so that there is no bifurcation to oscillating solutions for strings or squares. However, with these parameters there are bifurcations to oscillations in hexagons, but for much more negative values of $A$ than are shown on this scale. Longer range wavelengths (light shading) are predicted by the strongest activation of ligand production and the weakest for receptor production within the $\mathcal{A}, \mathcal{F}>0$ pattern region. For ligand inhibition, patterns of wavelength longer than 2 cells are also predicted in each geometry: at least 4 cells when $K=0$. 
(a) String
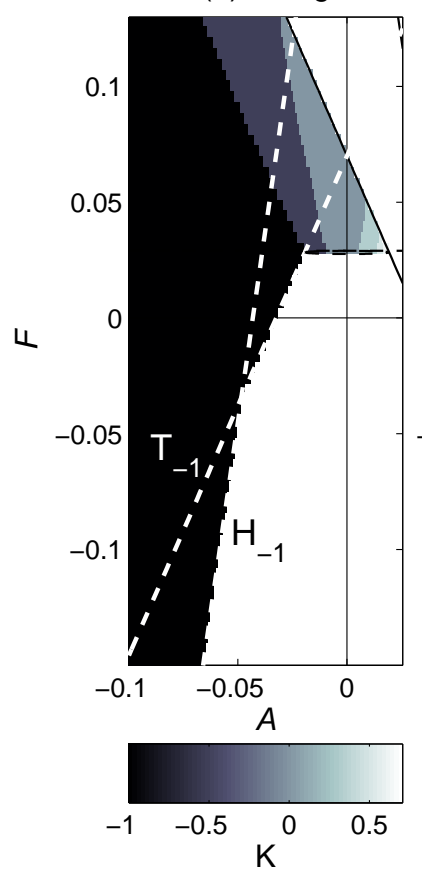

(b) Squares

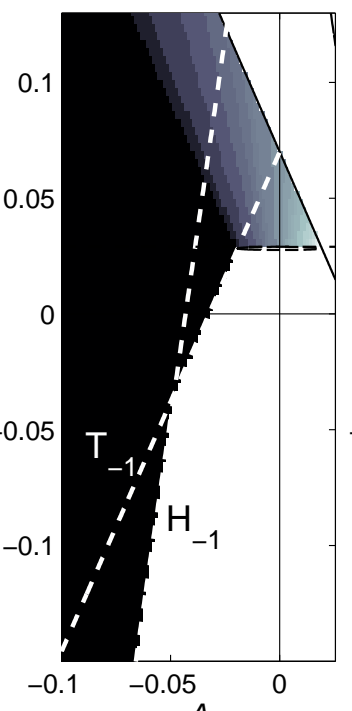

$A$

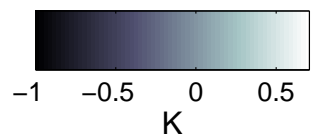

(c) Hexagons

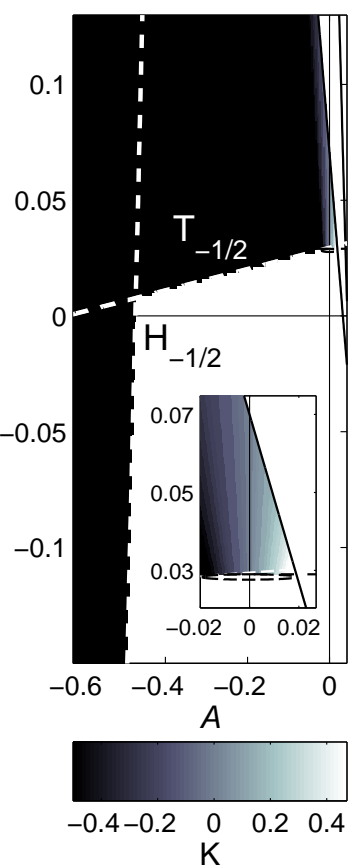

Fig. 10. An example of the variation of the fastest growing mode $K$ in the $(\mathcal{A}, \mathcal{F})$ space, with $d_{f}=0.01<0.03=d_{a}$ so that bifurcations to oscillatory patterns are possible: (a) strings, (b) squares and (c) hexagons. The simulation details and the remaining parameters are the same as in Figure 9. Linear analysis predicts oscillating patterns below the line $T_{\kappa}$ and above the line $H_{\kappa}$, where $\kappa=-1$ for strings/squares, and $\kappa=-1 / 2$ for hexagons. In each case the bifurcation to oscillating solutions is always for a wavelength of 2 cells in strings/squares and 3 cells in hexagons (shaded black). 
(a)

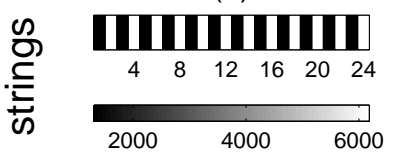

(d)

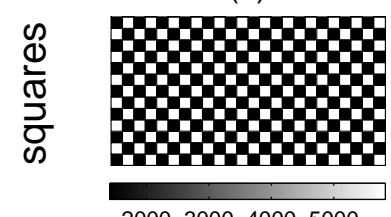

(g)

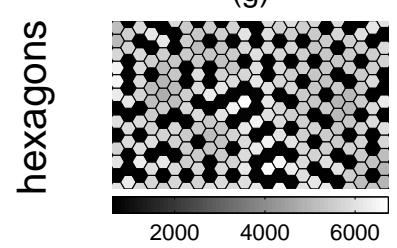

(b)

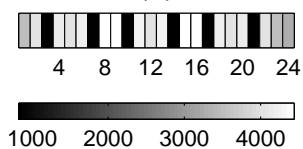

(e)

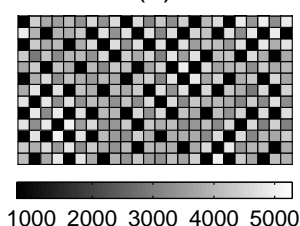

(h)

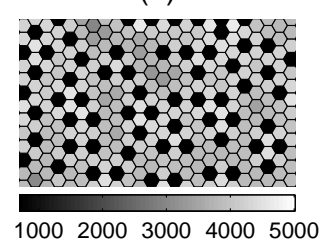

(c)

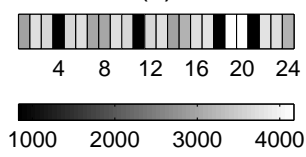

(f)

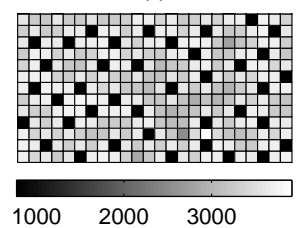

(i)

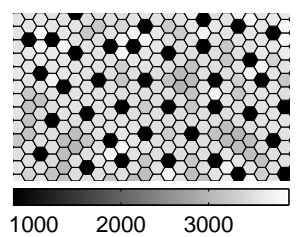

Fig. 11. Lateral inhibition gives rise to spatial patterns with wavelengths longer than 2 or 3 cells: nine different simulations of the juxtacrine model (1), solved on a string of 24 cells in (a-c), and a $24 \times 24$ array of square cells (d-f) and hexagonal cells (g-i). For clarity, in (d-i) we show a subset of the full $24 \times 24$ array. We plot the densities of bound receptors - ligand and free receptor densities have similar profiles. The initial conditions are small random perturbations about the homogeneous steady state. We take our boundary conditions to be periodic. Longer wavelengths with lateral inhibition in ligand production require sufficiently strong receptor responses $\left(F>T_{0}\right)$ and weaker ligand inhibition. The strongest ligand inhibition is shown in the left-hand column and the weakest in the righthand column. The parameter values are as given in Appendix B, except for $r_{0}=$ 1000, $\beta_{a}=0.3$, and with: (a) $m=-1.2, n=2$, (b) $m=-0.79, n=2.5$, (c) $m=-0.55, n=2.515$, (d) $m=-1.2, n=2$, (e) $m=-1, n=2.5$, (f) $m=-0.79$, $n=2.5$, (g) $m=-1.2, n=4$, (h) $m=-1, n=2.7$, and (i) $m=-0.79, n=2.5$. 
Table 1. The homogeneous steady state for (1) can lose stability to inhomogeneous perturbations when $a_{3}(K)=0$ or $\Delta(K)=0$, and stability to homogeneous perturbations requires $a_{3}(1)>0$ and $\Delta(1)>0$. Both $a_{3}$ and $\Delta$ are quadratic in $K$, so we analyse the nature and position of the critical point, $K_{\mathrm{c}}$, and roots of a general quadratic $Q(K)$. We subdivide the $(\mathcal{A}, \mathcal{F})$ plane into two regions: in region 1 bifurcations must correspond to a double root at $K_{\mathrm{c}} \in[\kappa, 1)$; in region 2 a patterning bifurcation occurs when $Q(\kappa)=0$. The regions are illustrated in Figure 3(a) for $a_{3}$ and Figure 4(a) for $\Delta$. This allows us to characterise the possible patterning bifurcations for strings/squares $(\kappa=-1)$, hexagons $(\kappa=-1 / 2)$, and stripes $(\kappa=0)$.

\begin{tabular}{|c|c|c|c|}
\hline Region & Max/Min & $Q(K)$ & Bifurcation \\
\hline 1 & $\begin{array}{l}\text { Min } \\
{[\kappa, 1)}\end{array}$ & & $Q\left(K_{\mathrm{c}}\right)=0$ \\
\hline \multirow[t]{3}{*}{2} & $\begin{array}{l}\text { Min } \\
\notin(\kappa,-1)\end{array}$ & & \multirow[t]{3}{*}{$Q(\kappa)=0$} \\
\hline & Max & & \\
\hline & & $k$ & \\
\hline
\end{tabular}

